\title{
A STUDY OF HADRONIC FINAL STATES
}

FROM INELASTIC MUON SCATTERING

\section{IN A HYBRID BUBBLE CHAMBER EXPERIMENT*}

J. Ballam, E. D. Bloom, J. T. Carroll, G. B. Chadwick, R. L. A. Cottrellt, M. Della Negrat+, H. DeStaebler, L. K. Gershwin+t+, L. P. Keller, M. D. Mestayer, K. C. Moffeit, C. Y. Prescott, S. Stein

Stanford Linear Accelerator Center Stanford University, Stanford, California 94305

\begin{abstract}
We present a preliminary analysis of the hadronic final states for inelastic scattering of $16 \mathrm{GeV}$ muons in the SLAC rapid cycling $40^{\prime \prime}$ hydrogen bubble chamber. From an exposure of 45,000 triggered pictures, representing approximately one-half of our data, we obtained 1500 inelastic events with $\mathrm{Q}^{2}>0.15(\mathrm{GeV} / \mathrm{c})^{2}$. Results for charged hadron multiplicities, inclusive proton, $\pi^{+}$, and $\pi^{-}$spectra, $\rho^{\circ}$ and $\omega$ final states, and charge ratios are given.
\end{abstract}

(XVI International Conference on High Energy Physics

Chicago and Batavia, Illinois, September 1972)

\footnotetext{
* Work supported by the U.S. Atomic Energy Commission.

+ Presently on leave at CERN.

+ On leave from College de France, Paris.

++ Present address: Institute for Defense Analyses, Washington, D.C.
} 


\section{INTRODUCTION}

In this paper we report on a preliminary analysis of a hybrid bubble chamber experiment examining the process $\mu^{-}+p \rightarrow \mu^{-}+X$ where $X$ is detected with $4 \pi$ geometry.

The general kinematics of the process is as follows:

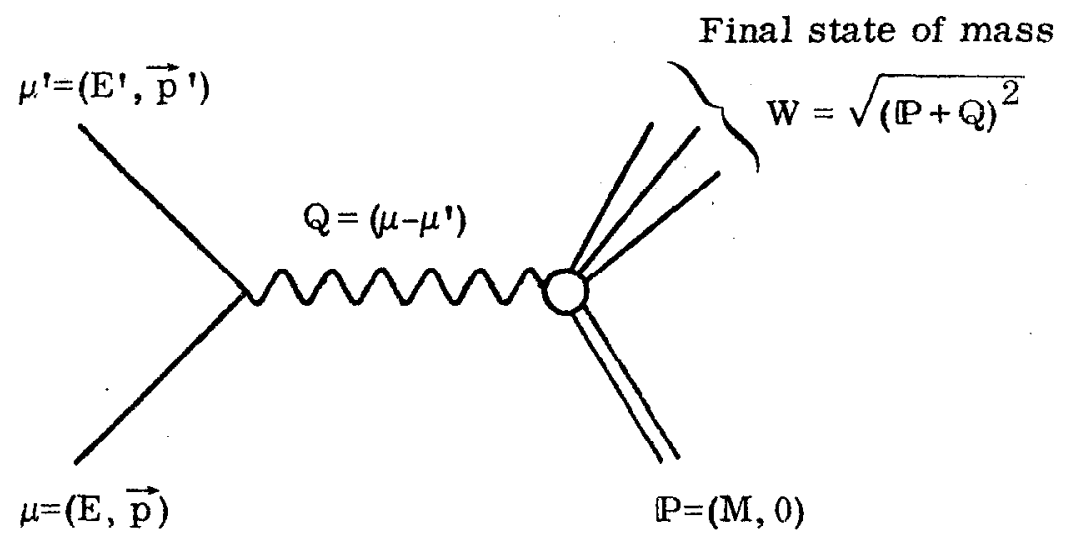

where conventionally we define,

$$
\begin{aligned}
& Q^{2}=-\left(\mu-\mu^{\prime}\right)^{2}=-2 m_{\mu}{ }^{2}+2\left(E E^{\prime}-|\vec{p}|\left|\vec{p}^{\prime}\right| \cos \theta\right) \\
&=Q_{\text {min }}^{2}+4|\vec{p}|\left|\vec{p}^{\prime}\right| \sin ^{2} \theta / 2>0 \\
& Q_{\min }^{2}=2\left(E E^{\prime}-|\vec{p}||\vec{p} \cdot|-m_{\mu}^{2}\right) \\
& \nu=\frac{\mathbb{P} \cdot Q}{M}=E-E^{\prime}, \quad M=\text { mass of proton } \\
& s=W^{2}=2 M \nu+M^{2}-Q^{2}
\end{aligned}
$$

Note that $Q^{2}, \nu$ and $s$ are Lorentz invariants and $(E, \vec{p}),\left(E^{\prime}, \vec{p}^{\prime}\right)$ are the incoming and scattered muon energy and momentum in the laboratory frame while $\theta$ is the laboratory scattering angle of the muon. 
The double differential cross section for muon detection is usually expressed in two ways.

$$
\begin{aligned}
& \frac{\mathrm{d}^{2} \sigma}{\mathrm{dQ}^{2} \mathrm{ds}}=\left(\frac{\pi}{2 M E E^{1}}\right) \cdot \sigma_{M}\left[W_{2}\left(s, Q^{2}\right)+2 \tan ^{2} \theta / 2 W_{1}\left(s, Q^{2}\right) I\right. \\
& \text { where } \sigma_{M}=\frac{4 \alpha^{2} E^{\prime 2} \cos ^{2} \theta / 2}{Q^{4}}
\end{aligned}
$$

or

$$
\frac{d^{2} \sigma}{d Q^{2} d s}=\left(\frac{\pi}{2 M E E^{\prime}}\right) \quad \Gamma_{t}\left[\sigma_{T}\left(s, Q^{2}\right)+(\epsilon+\delta) \sigma_{S}\left(s, Q^{2}\right)\right]
$$

where

$$
\begin{aligned}
& \Gamma_{\mathrm{t}}=\frac{\alpha}{4 \pi^{2}} \frac{\mathrm{W}^{2}-\mathrm{M}^{2}}{\mathrm{MQ}} \frac{\mathrm{E}^{\prime}}{\mathrm{E}(1-\epsilon)} \\
& \epsilon=\frac{1}{1+\frac{2\left(\mathrm{Q}^{2}+\nu^{2}\right) \tan ^{2} \theta / 2}{\mathrm{Q}^{2}\left(1-\mathrm{Q}_{\min }^{2} / \mathrm{Q}^{2}\right)^{2}}} \\
& \delta=\frac{2 \mathrm{~m}_{\mu}^{2}}{\mathrm{Q}^{2}}(1-\epsilon)
\end{aligned}
$$

And the factor $\left(\pi / 2 M E E^{\prime}\right)$ converts $d^{2} \sigma / d \Omega d E^{\prime}$ to $d^{2} \sigma / d Q^{2} d s$. In our kinematical range $Q_{\min }^{2}$ and $\delta$ are negligible. $W_{1}\left(s, Q^{2}\right)$ and $W_{2}\left(s, Q^{2}\right)$ are the structure functions of the proton, in general functions of both $s$ and $Q^{2}$. The single arm inclusive ep inelastic scattering measurements done at SLAC $^{(1)}$ over the past five years have shown that $\nu \mathrm{W}_{2}$ scales, i.e., $\nu \mathrm{W}_{2}=\mathrm{F}\left(\mathrm{s} / \mathrm{Q}^{2}\right)$ if $\mathrm{Q}^{2} \gtrsim 1(\mathrm{GeV} / \mathrm{c})^{2}$ and $s \gtrsim 4 \mathrm{GeV}^{2}$, and so the use of the structure function representation has become particularly common. However, the second representation, where $\sigma_{\mathrm{T}}$ and $\sigma_{\mathrm{S}}$ are the total cross sections for transversely and longitudinally polarized photons respectively, is more revelant to our presentation here, 
since comparisons with photoproduction are quite instructive, and $\sigma_{\mathrm{T}}\left(\mathrm{s}, \mathrm{Q}^{2}\right) \rightarrow \sigma_{\gamma \mathrm{p}}(\mathrm{s})$ as $\mathrm{Q}^{2} \rightarrow 0$. (From its definition $\nu \mathrm{W}_{2} \rightarrow 0$ as $\mathrm{Q}^{2} \rightarrow 0$.) Also, much of our data lies in the transition region between photoproduction and the scaling region and so expressing our results in terms of $\nu \mathrm{W}_{2}$ does not seem particularly revealing at this time.

Figure 1 shows the inclusive ep inelastic cross section ${ }^{(2)}$ at $W=3$ to $5 \mathrm{GeV}$ as a function of $\mathrm{Q}^{2}$, from photoproduction to $\mathrm{Q}^{2}=1.5(\mathrm{GeV} / \mathrm{c})^{2}$, approximately the $\mathrm{Q}^{2}$ range of this experiment. The decrease of the $\sigma_{\gamma_{V}}^{\text {tot }}$ with $\mathrm{Q}^{2}$ may represent a rapidly changing transition region between photoproduction and the scaling region, with most of the transition completed by a $Q^{2} \gtrsim 0,6(\mathrm{GeV} / \mathrm{c})^{2}$. This feature of the ep inclusive data was a strong motivation for this experiment which attempts to investigate largely qualitative features of the inelastic final states with limited statistics using the unbiased $4 \pi$ geometry of the bubble chamber.

The study of the final states of $\mu$ p scattering poses somewhat of a dilemma to the experimentalist. The intrinsically small total cross sections, less than $1 \mu \mathrm{b}$ for all $\mathrm{Q}^{2}>0.1(\mathrm{GeV} / \mathrm{c})^{2}$, suggests using countercoincidence techniques to study the final states. However, the scarcity of knowledge of the nature of the final states suggests an unbiased initial investigation to determine major trends and features in the process. The bubble chamber is an obvious instrument to use as an unbiased $4 \pi$ detector, but conventional bubble chamber techniques would require about 100 million piciures for a few thousand events.

One solution to the dilemma is to use a bubble chamber in a triggered "hybrid" mode. By placing a $\mu$-telescope behind the SLAC $40^{\prime \prime}$ bubble chamber one can determine with high probability when an interaction of interest has occurred in the chamber. Thus a bubble chamber picture is taken, i.e., the 
bubble chamber lights flashed, only when the $\mu$-telescope indicates the presence of an event of interest. This procedure yielded about 5,000 inelastic plus elastic events in 94,000 pictures with 30 million expansions of the bubble chamber.

Other improtant differences should be noted between this experiment and more conventional exposures. Firstly, at normal bubble chamber rates of $\sim 1$ expansion per second, about 8 thousand hours of data taking are required to obtain 30 million pulses. Happily, the SLAC bubble chamber operations group has been working on rapid cycling techniques with the $40^{\prime \prime}$ hydrogen bubble chamber over the past few years, and so this experiment was run with the bubble chamber pulsing at 10 expansions per second for over $90 \%$ of our datataking time. Secondly, due to the small probability of a given $\mu$ interacting, we were able to run typically with more than $100 \mu$ 's/pulse at $16 \mathrm{GeV} / \mathrm{c}$ through the chamber. This is another factor of 10 over typical hadronic beam exposures.

\section{EXPERIMENTAL SETUP}

\section{A. $\mu$-Beam}

In $\mathrm{Fig} .2$ is shown a schematic representation of the experiment from the origin of the muon beam to the muon telescope behind the bubble chamber. Muons were pair produced in the first few radiation lengths of a high $\mathrm{Z}$ targetbeam dump, by a $20 \mathrm{GeV} \mathrm{e}^{-}$beam of $40 \mathrm{~mA}$ instantaneous current (pulse length $1.5 \mu \mathrm{sec})$. The $\mu$ beam cons isted of four stages with three intermediate foci. The momentum analysis was done in the first stage by means of a $30 \mathrm{~cm}$ long tungsten slit placed at the first focus defining a momentum bite of $\Delta p / p= \pm 2 \%$. At this focus the muons have to pass through $3.7 \mathrm{~m}$ of beryllium (approximately 7.5 interaction lengths) which filters out hadrons produced in the thick target.

$$
-5-
$$


The measured $\pi$ to $\mu$ ratio in the beam after the beryllium filter was $(.53 \pm .10)$

$\times 10^{-4}$. The increase of phase space due to Coulomb scattering in the filter was kept to a minimum by placing the filter at a focus.

The purpose of the next three stages after the filter was to reduce the bearn halo. At each intermediate focus (focus 1 and focus 2 ) the muons outside the beam envelope were scattered out by a $2 \mathrm{~m}$ long lead collimator. Each collimator was followed by about $10 \mathrm{~m}$ of iron to stop the $\mu$ 's scattered out of the beam envelope. The amount and shape of shielding was designed using a computer simulation of the beam so as to further reduce the halo. The energy of the beam after the Be filter, $16 \mathrm{GeV}$, was chosen to be the maximum value compatible with the requirement of having about $100 \mu$ 's per pulse at the present energy and intensity of the SLAC $\mathrm{e}^{-}$beam.

Proportional wire chambers placed at foci 1 and 2 and in front of the bubble chamber were used to study beam profiles, correlations between different foci, and measure beam halo.

The measured parameters of the beam at the bubble chambers are summarized below:

\section{TABLE I}

Momentum

Momentum bite

Beam intensity

Vertical beam size

Horizontal beam size

Vertical angular divergence

Horizontal angular divergence

Penetrating halo
$16 \mathrm{GeV} / \mathrm{c}$

$7.4 \%$

FWHM

$100 \mu /$ pulse

$11 \mathrm{~cm}$

FWHM

$1.2 \mathrm{~cm}$

FWHM

$4 \mathrm{mrad}$

FWHM

$3.5 \mathrm{mrad}$

FWHM

$2 \%$

$-6-$ 


\section{B. Bubble Chamber}

The bubble chamber is a $1 \mathrm{~m}$ diameter cylinder, $0.4 \mathrm{~m}$ deep with a vertical glass window and scotchlite illumination. Expansion is by means of a bellows mounted to a piston the full width of the chamber which is hydraulically actuated. No degradation of measuring accuracy was observed at 10 expansions per second -- the setting error was about 150 microns in space. Aside from increased failures of elements of the expansion system compared to lower rate running, no major mechanical problems were encountered. Loss of time from bubble chamber failures after the major development to 10 expansions per second was completed was comparable to previous low rate running.

\section{C. $\mu$ Telescope}

Behind the bubble chamber was placed an apparatus which selectively triggered on muons with a scattering angle $\gtrsim 1.4$ degrees. This device consisted of two sets of coarse scintillation hodoscopes in $x$ and $y$ (see Fig. 2), in a sandwich of four 12 inch iron blocks and eleven $1 \mathrm{~m} \times 1 \mathrm{~m}$ magnetostrictive spark chambers.

With the veto counters in front of the bubble chamber the trigger counter hodoscopes defined a fast logic trigger which initiated the spark chambers and interrupted the on-line PDP-8L computer. At this point all the hodoscopes were strobed into the computer and a simple 0.5 millisecond calculation was done to accept only hodoscope patterns consistent with particle trajectories coming from the bubble chamber fiducial volume. If the computation was satisfactory the bubble chamber lights were flashed, a picture taken, and the spark chamber and other event information was written on magnetic tape. This procedure yielded an event and picture rate as indicated in Table $\Pi$.

In addition to its use as a trigger for the bubble chamber lights, the muon 
telescope behind the bubble chamber served three other purposes: i) With a pion contamination of $\lesssim, 53 \times 10^{-4}$ as described above, roughly equal numbers of muon and pion interactions occur in the bubble chamber. We estimate that the muon telescope reduced this contamination in our final event sample to $<0.5 \%$ averaged over the full kinematic range. ii) Particularly in many prong events, the prong which was the muon was impossible to determine using bubble chamber information only. The muon telescope thus was used to "match" the muon in the bubble chamber with the penetrating trigger track in the spark chambers. iii) The magnetic field of the SLAC $40^{\prime \prime}$ bubble chamber has $\int \mathrm{Bdl}=26 \mathrm{kG}-\mathrm{m}$ between the center of the chamber and the position of the muon telescope. In our analysis we used this field in conjunction with the muon telescope to improve the precision of measurement on the outgoing muon momentum to about $\pm 200 \mathrm{MeV} / \mathrm{c}$, making separation of events with a singlemissing neutral from multi-missing neutrals possible in this experiment. 


\section{TARLE II}

$\underline{\text { Event Rate }}$

1 hardware trigger in 15,000 tracks

1 computer trigger in 32,000 tracks

1 candidate* $^{*}$ in 11 computer triggers

1 accepted* event in 16 computer triggers

At $100 \mu^{\prime}$ s per pulse:

1 picture in 320 chamber expansions

1 accepted event in 5120 chamber expansions

At 10 expansions per second:

120 pictures per hour

7.5 accepted events per hour

$70 \%$ of these are inelastic events, the rest are elastic. To get

4000 inelastic events,

30 million expansions

3000 million $\mu^{\prime} \mathrm{s}$

With the aid of a trigger, there are about 94,000 pictures.

* See Section III for appropriate definitions.

\section{BUBBLE CHAMBER ANALYSIS}

The data presented in this report is taken from an analysis of 45,000 pictures, or approximately half our film. A typical event picture is shown in Fig. 3.

The first step in the film processing was a scan for candidate events to be measured. A candidate was defined to be an interaction with a positive prong 
occurring within the $33.1^{\prime \prime}$ fiducial volume of the $40^{\prime \prime}$ bubble chamber. The film was scanned twice, once by the physicists, and discrepancies between the two scans were resolved by a third conflict scan. This procedure yielded a calculated scanning efficiency of $99 \%$. An additional, independent check of the scanning efficiency was made by scanning a third time those frames for which the patterns of sparks in the spark chambers and fired trigger counters satisfied our criteria for a good $\mu$ track, but in which no interaction had already been found. About $20 \%$ of the pictures fell in this category, and the third scan yielded an additional $0.8 \%$ accepted events.

All candidates were measured on conventional film plane digitizers. Ninety-six percent of the candidates had good measurements on all tracks after three measurement passes. The candidates were analyzed using a modified TVGP-SQUAW reconstruction and kinematic fitting programs. For each candidate the physicists checked the track ionization and selected those fit hypotheses compatible with the observed bubble density. Protons could be resolved from pions up to about $1 \mathrm{GeV} / \mathrm{c}$. The $\mu^{-}$match between the bubble chamber and spark chambers was also checked for each event by the physicists. Candidates which did not yield a good $\mu^{-}$match with a penetrating, triggering track in the spark chambers were not used in this analysis. This procedure yielded 2600 accepted elastic and inelastic scatters (accepted events) from a sample of more than 3700 candidates.

The large fiducial volume of 33.1 " used in this analysis was achieved by using the spark chambers downstream from the bubble chamber and the fringe field of the bubble chamber magnet to obtain a more accurate measurement of the scattered $\mu^{-}$direction and momentum. With $12 \mathrm{~cm}$ of track in the bubble chamber we could determine the $\mu^{-}$momentum to typically $4 \%$ using the spark chamber data. 
IV. RESULTS

\section{A. Kinematical Distribution of the Data}

In Fig. 4 we show the extent of our data in a scatter plot of $Q^{2}$ vs $W$. Elastic events are shown clearly on the left. Inelastic events extend to $\mathrm{W}=5 \mathrm{GeV}$ and to $Q^{2}=2.5(\mathrm{GeV} / \mathrm{c})^{2}$. On Fig. 4a we show contours of our detection probability $\mathrm{P}$ for scattered muons. These are obtained from a Monte Carlo calculation and are typically greater than 0.7 in our data sample. In Fig. $4 \mathrm{~b}$ we show the same data with contours of $\epsilon$, the ratio of longitudinal to transverse polarization for the virtual photon, and $\omega^{\prime}=1+\mathrm{W}^{2} / \mathrm{Q}^{2}$, the scaling variable.

\section{B. Total Cross Section and Topological Partial Cross Sections}

The total cross section, $\sigma_{\mathrm{T}}+\epsilon \sigma_{\mathrm{S}}$, is related to the experimental cross section by the flux factor $\Gamma_{t}$ according to $\mathrm{Eq} .7$.

In order to extract an experimental cross section, the accurate monitoring of incident muon flux was necessary. This was achieved by means of a scintillation counter placed in the beam. Direct counting of the muons in the beam was not possible, because the rates were too high (approximately 100 muons $/ 1.5$ $\mu$ sec pulse) for fast electronics to work accurately. Instead, the scintillation signal was first integrated, then digitized and accumulated in a scaler. The calibration was set to give approximately 1 count/incident $\mu$. Frequent and accurate calibration of the beam monitor was obtained by comparison of the monitor output with a direct count of $\mu$-tracks in the bubble chamber. The error in the incident flux was estimated to be $\leq \pm 5 \%$.

A Monte Carlo calculation determines for us the probability of detecting a scattered muon. This probability depends only on the $\mathrm{Q}^{2}$ and $\nu$ values for the muon, since the triggering scheme does not require the presence of hadrons 
in the final state. The dependence on the position of the vertex in the bubble chamber is removed by appropriately averaging over the intersection of the beam with the bubble chamber fiducial volume. For the cross section values shown, the probability for detecting a scattered $\mu$ varies from 0.3 to 1.0 and is typically 0.7 (see Fig. 4a).

The resulting values for $\sigma_{\mathrm{T}}+\epsilon \sigma_{\mathrm{S}}$ are shown in Figs. 5a-5c, We estimate an overall systematic uncertainty on the order of $\pm 10 \%$ for these values. For most of the data considered (with the exception of photoproduction points), $\epsilon$ varies from 0.8 to 1.0 , (see Fig $4 \mathrm{~b}$ ). From our data $\sigma_{\mathrm{T}}$ and $\sigma_{\mathrm{S}}$ cannot be separated in a model independent way.

Three $W$ bins are considered: $W=1.4$ to $1.8(\mathrm{Fig}, 5 \mathrm{a}), \mathrm{W}=1.8$ to $2.8 \mathrm{GeV}$ (Fig. $5 \mathrm{~b}$ ), and $\mathrm{W}=2.8$ to $3.8 \mathrm{GeV}$ (Fig. $5 \mathrm{c}$ ). Each cross section point is broken down into its charged multiplicity. Only charged hadrons contribute to the prong count. The ratio of 1-prongs to $\sigma_{\mathrm{TOT}}$ (observed value) and the ratio of 3 -prongs to $\sigma_{\text {TOT }}$ are given at each $Q^{2}$ for the points in $\mathrm{F}^{\mathrm{i}} \mathrm{ig}$. $5 \mathrm{a}$. In Fig. 5b, 5-prongs also contribute to the total cross section and in $5 c$, also 7-prongs.

The general features show agreement, within systematic errors, between $\mu$-beam total cross sections and SLAC single arm electroproduction values. ${ }^{(2)}$ At $\mathrm{W}=1.4-1.8 \mathrm{GeV}$, the single prong contribution to $\sigma_{\mathrm{TOT}}$ is consistent with a constant value of about 0.7 , and the 3 -prongs contribute the rest of $\sigma_{\text {TOT }}{ }^{*}$ The photoproduction points are obtained from ref. 3. In $F$ ig. $5 b$, at $W=1.8$ to $2.8 \mathrm{GeV}$, we see an increasing fraction of the total cross section in the 1 -prong topology, as $Q^{2}$ increases, and this increase is reflected in a corresponding decrease in the 3-prong topology, while 5-prongs contribute a small amount. The photoproduction values in Figs. $5 \mathrm{~b}$ and $5 \mathrm{c}$ are obtained from data of ref. 4 . 
In Fig. 5d we present the 1-prong data of Fig. 5b again, and show the contribution to the 1-prongs that is consistent with a single missing neutral hypothesis ( $1 \mathrm{C} \mathrm{fit})$. For this topology, the $1 \mathrm{C}$ hypotheses are:

$$
\begin{aligned}
& \gamma_{\mathrm{v}} \mathrm{p} \rightarrow \mathrm{p} \pi^{0} \\
& \gamma_{\mathrm{V}} \mathrm{p} \rightarrow \pi^{+} \mathrm{n} .
\end{aligned}
$$

Generally, an event satisfying one of the hypotheses will also satisfy the other hypothesis, unless one can be selected on the basis of ionization. For these data typically $50 \%$ of the $1 \mathrm{C}$ fits satisfied both hypotheses and, therefore, no attempt has yet been made to separate the two. The points shown, therefore, represent an estimate of the contribution of $\pi \mathrm{N}$ final states to the total cross section. The rest of the 1-prong contribution is associated with multiple neutrals in the final state. We also include corresponding photoproduction points. The value for $0\left(1-\right.$ prong) $/ \sigma_{\text {TOT }}$ at $Q^{2}=0$ comes from ref. 4 , while the value for $\sigma\left(\pi^{+} \mathrm{n}+\pi^{o} \mathrm{p}\right) / \sigma_{\text {TOT }}$ at $\mathrm{Q}^{2}=0$ is obtained by integrating differential cross section values from a compilation of high energy photoproduction data.

\section{Multiplicities}

One may summarize the prong distributions shown in Fig. 5 by computing $<N>$, the mean charged hadronic prong multiplicity for various bins in $Q^{2}$ and $W$.

$$
\langle\mathrm{N}\rangle=\left(\sum_{\mathrm{i}} \mathrm{N}_{\mathrm{i}} / \mathrm{P}_{\mathrm{i}}\right) /\left(\sum_{\mathrm{i}} 1 / \mathrm{P}_{\mathrm{i}}\right)
$$

where $N_{i}$ is the number of prongs in an event and $P_{i}$ is the acceptance probability for the event derived from the Monte Carlo calculations mentioned above. Figure 6 a shows $\left\langle N>\right.$ vs $Q^{2}$ for three $W$ ranges. There is a tendency for $<\mathrm{N}>$ to decrease as $\mathrm{Q}^{2}$ increases, particularly for $\mathrm{W}=1.8-2.8 \mathrm{GeV}$ where the effect clearly arises from the relative decrease in 3-prong events and the 
corresponding increase in 1-prong events.

The multiplicity was also studied as a function of $Q^{2}$ in the $W$ range $2-4 \mathrm{GeV}$ in the photon fragmentation region $(x>0.2)$, the target fragmentation region $(x<-0.2)$, and the central region. To do this we binned the charged hadrons from each event into their proper $Q^{2}$ and $x$ regions as shown in Fig. 6b, where $x=\left(p_{\|} / p_{\text {max }}\right) \mathrm{cms}$. In the case of events in which the positive track identification was ambiguous, the pion hypothesis was selected rather than the proton. Choosing the proton hypothesis for the track gives an $\mathbf{x}$-value more negative than that for the pion because of the mass dependence of the Lorentz transformation to the cms. The photoproduction data were handled in the same way. ${ }^{(9)}$ The central region multiplicity is consistent with a flat dependence on $\mathrm{Q}^{2}$, while the target and projectile region multiplicities seem to be falling slightly with $Q^{2}$.

Although the purely statistical evidence is fairly strong in favor of $\langle\mathrm{N}\rangle$ decreasing as $Q^{2}$ increases, we want to emphasize that our whole analysis is preliminary, that no effects of possible systematic errors in our experiment are included, and that these conclusions depend to a large extent on the comparison of the present experiment with the photoproduction experiment where there might be additional relative systematic errors.

D. $e^{0}$ Production

Events of the reaction

$$
\mu^{-} \mathrm{p} \rightarrow \mu^{-} \mathrm{p} \pi^{+} \pi^{-}
$$

could be clearly isolated by requiring a good $4 \mathrm{C}$ fit. The invariant mass of dipions in this sample, for $2.0<\mathrm{W}<4.0 \mathrm{GeV}$, is shown in Fig. 7 a where a clear $\rho^{\circ}$ signal is evident. For $\mathrm{W}<2.0 \mathrm{GeV}$ the signal is indistinct and so only the higher energy data were used in this analysis. We note that the mean 
acceptance weight varies by less than $20 \%$ across the plot. Superimposed on the mass spectrum in Fig. 7a, is the approximate behavior of the $2.8 \mathrm{GeV}$ photoproduction data of the SLAC-LBL-Tufts collaboration ${ }^{(4)}$ normalized to the data in the $\rho^{\mathrm{o}}$ regions $\left(0.65<\mathrm{M}_{\pi \pi}<0.9 \mathrm{GeV}\right)$; the mean $\mathrm{W}$ is approximately the same in the two cases.

We estimate that the non-rho background is about $10 \%$ of all dipions with $0.65<\mathrm{M}_{\pi \pi}<0.90 \mathrm{GeV}$, and the $\rho^{\mathrm{O}}$ events lost outside this mass cut are also about $10 \%$. Hence we refer to the cross section for dipions in the above mass cut as $\left(\rho^{\circ}\right)$. The total $\rho^{\circ}$ cross section normalized to $\sigma_{\text {TOT }}\left(W, Q^{2}\right)$ is shown in Fig. $7 \mathrm{~b}$ for our data. The point at $\mathrm{Q}^{2}=0$ was obtained by averaging the $\rho^{\mathrm{O}}$ and total photoproduction cross sections over our virtual photon flux, using the data of the $\mathrm{SWT}^{(5)}$ and $\mathrm{SBT}^{(4)}$ collaborations, which were deduced using the Söding Model. ${ }^{(6)}$ The relative importance of $\rho^{0}$ photoproduction apparently decreases with $Q^{2}$ by about a factor of 2 over our $Q^{2}$ range. We would expect some decrease in $\sigma_{\rho}$ due to the $\mathrm{t}_{\min }$ effect; i.e., the minimum momentum transfer to the proton increases with increasing $Q^{2}$ over that of photoproduction at the same W. If we assume that $\frac{\mathrm{d} \sigma}{\mathrm{dt}}\left(\mathrm{W}, \mathrm{Q}^{2}\right)$ is equal to $\frac{\mathrm{d} \sigma}{\mathrm{dt}}(\mathrm{W}, \mathrm{O})$ at all $\mathrm{Q}^{2}$, then the low $t$ region will be kinematically excluded. We find that this effect would give reduction in the total $\rho^{\circ}$ cross section of $10 \%$ and $30 \%$, respectively, in the two highest $\mathrm{Q}^{2}$ bins in Fig. 6, assuming a dependence in $\mathrm{d} \sigma / \mathrm{dt}$ of $\mathrm{e}^{6.8 \mathrm{t}}$.

The $t$ dependence of $\rho^{\circ}$ production by $\mu$ 's is illustrated in Fig. 8 . In the interval $0.05<|\mathrm{t}|<0.6 \mathrm{GeV}^{2}$, the slope found for a fit to the form $\mathrm{e}^{\mathrm{Bt}}$ is $7.1 \pm 1.9 \mathrm{GeV}^{-2}$ for $0.15<\mathrm{Q}^{2}<0.5 \mathrm{GeV}^{2}$ and $4.9 \pm 1.3 \mathrm{GeV}^{-2}$ for $0.5<\mathrm{Q}^{2}$ $<2 \mathrm{GeV}^{2}$. If $\mathrm{t}^{\prime}=\mathrm{t}-\mathrm{t}_{\mathrm{min}}$ is used, with the same assumption, the fitted slopes for $0.0 \leq|\mathrm{t}| \leq 0.6(\mathrm{GeV} / \mathrm{c})^{2}$ are not significantly changed $(7.5 \pm 1.6$ and $4.9 \pm 1.4 \mathrm{GeV}^{-2}$ are respectively the slopes). There is, therefore, marginal 
evidence ( $\sim 2$ S. D.) that the $\rho^{\circ}$ slope decreases with increasing $Q^{2}$, as was reported in coincidence electroproduction experiments. ${ }^{(12)}$ The broken line in Fig. 8 is proportional to $\mathrm{e}^{5.6 \mathrm{t}}$, where $5.6 \mathrm{GeV}^{-2}$ is the slope found, using the Soding Model analysis, for $2.8 \mathrm{GeV} \rho^{\circ}$ photoproduction by the SBT collaboration. ${ }^{(4)}$ with the present data they are also compatible with the photoproduction slope. We must note here, however, that a comparison of these slopes with photoproduction may well be misleading, because there can be different incoherent backgrounds present in the two cases (see ref. 4 for discussions).

For the description of the $\rho^{\circ}$ decay when produced in the inelastic $\mu$ scattering, the usual density matrix representation ${ }^{(7)}$ must be expanded to include production by longitudinal photons. If $\theta$ and $\phi$ are the polar and azimuthal angles of the $\pi^{+}$in the $\rho^{\circ}$ rest system (with the $\mathrm{z}$ axis along the cms $\rho^{\circ}$ direction, the $\mathrm{x}$ axis in the hadron production plane, and $\Phi$ the azimuth of the scattered $\mu$ with respect to the hadron production plane in the overall cms) (4)

$$
\begin{array}{r}
\mathrm{W}(\theta, \phi, \Phi) \sim \mathrm{W}^{0}-\epsilon \cos 2 \Phi \mathrm{W}^{1}-\epsilon \sin 2 \Phi \mathrm{W}^{2} \\
-\mathrm{p} \cos \Phi \mathrm{W}^{3}-\mathrm{p} \sin \Phi \mathrm{W}^{4}
\end{array}
$$

where $\epsilon$ was defined previously in Eq. 9 and

$$
p=\sqrt{\epsilon(1+\epsilon+2 \delta)} \text {. }
$$

The $\mathrm{W}^{\alpha}$ are the same as for unpolarized transverse photons except

$$
\rho_{\mathrm{ik}}^{\mathrm{o}} \sim \rho_{\mathrm{ik}}^{\mathrm{oT}}+2(\epsilon+\delta) \rho_{\mathrm{ik}}^{\mathrm{oS}}
$$

Where $\mathrm{T}$ and $\mathrm{S}$ refer to production by transverse and longitudinal photons respectively and $\delta$ was defined in Eq. 10. It is clear that $\rho^{\text {oT }}$ and $\rho^{\text {oS }}$ can only be separated by varying $\epsilon+\delta$; for our data no separation is possible. 
In Fig. 9 we show the angular distribution of $\rho^{0}$ decay for $0.15<Q^{2}<2(\mathrm{GeV} / \mathrm{c})^{2}$ and $2<W<4 \mathrm{GeV}$. The data are consistent with isotropy in $\cos \theta$. The peak at $\cos \theta \approx-1.0$ is due to a small contamination from $\Delta^{++} \pi^{-}$. The value for $\rho_{00}^{0}$ was found from a moment analysis to be $0.2 \pm 0.1$ where we corrected for the $\Delta^{H+}$ contamination by using the Eberhard-Pripstein procedure. (14) If we assume that the $\rho^{0}$ production mechanism still conserves $s$-channel helicity when $Q^{2} \neq 0$, then $\rho_{00}^{0}$ measures $\sigma_{\mathrm{S}}(\rho) / \sigma_{\mathrm{T}}(\rho)=\rho_{00} /\left(1-\rho_{00}\right)$. The value found indicates that $\sigma_{S}(\rho)=0.25_{-0.16}^{+0.25} \sigma_{T}(\rho)$

The distribution in the angle $\Psi=\phi-\Phi$, which was found to show the greatest effect in $\rho^{\circ}$-photoproduction (i. e., $\sim \cos ^{2} \Psi$ ) by linearly polarized photons, is alsn shown in Fig. 9. The effect is still present but clcarly reduced, probably because of the presence of longitudinally polarized $\rho^{\circ}$.

\section{E. $\omega$ Production}

In Fig. 10a we plot $\mathrm{M}\left(\pi^{+} \pi^{-} \pi^{\circ}\right)$ from the reaction $\mu^{-} \mathrm{p} \rightarrow \mu \mathrm{p} \pi^{+} \pi^{-} \pi^{\circ}$, selecting only unambiguous protons $\left(|t|<1 \mathrm{GeV}^{2}\right)$ The events shown have a $1 \mathrm{C}$ fit confidence level greater than $10 \%, \mathrm{Q}^{2} \geq 0.15(\mathrm{GeV} / \mathrm{c})^{2}$ and no cut on W. We observe a clear $\omega$ peak. The shaded events have $Q^{2} \geq 0.5(\mathrm{GeV} / \mathrm{c})^{2}$ and may also show a $\omega$ peak. The events plotted have not been weighted by our acceptance; however, such weighting makes little change in the observed structure. We have estimated the $\omega$ cross section by selecting events with $0.74<\mathrm{M}(3 \pi)<0.82 \mathrm{GeV}$, making a small background correction by hand, and correcting for our $10 \% \chi^{2}$ probability cut and non $3 \pi$ decay modes. In $\mathrm{Fig}$. $10 \mathrm{~b}$ we plot the ratio $\sigma\left(\gamma_{\mathrm{v}} \mathrm{p} \rightarrow \mathrm{p} \omega\right) / \sigma_{\text {TOT }}$ vs. $\mathrm{Q}^{2}$ for $1.8<\mathrm{W}<3.8 \mathrm{GeV}$. The average value of $\mathrm{W}$ for all events in this plot, i.e., all $\mathrm{Q}^{2}$ bins, is $2.26 \mathrm{GeV}$. Using photoproduction data $^{(8)}$ we have calculated the ratio $\sigma(\omega) / \sigma_{\text {TOT }}$ at $\mathrm{Q}^{2}=0$ for the same $\mathrm{W}$ interval (correcting for the photon energy spectrum). The photoproduction value 
agrees well with our lowest $Q^{2}$ bin. Our highest $Q^{2}$ bin contains one $\omega$ event. Figure $10 \mathrm{~b}$ indicates that the $\omega$ remains a relatively small fraction of the cross section as $Q^{2}$ increases.

\section{F. Inclusive Distributions}

We present the inclusive distributions for

$$
\begin{aligned}
& \gamma_{v} p \rightarrow \pi^{ \pm}+(\text {anything }) \\
& \gamma_{v} p \rightarrow p+\text { (anything) }
\end{aligned}
$$

in the $Q^{2}$ and $W$ intervals shown and compare these data to photoproduction results. ${ }^{(9)}$ The differential cross section for such reactions can be written with the detected particle phase space explicitly shown:

$$
d^{2} \sigma=\pi \frac{p^{*} \max }{E^{*}} \operatorname{dxdp}_{1}^{2} \text { f }\left(x, p_{1}^{2}, s, Q^{2}\right)
$$

The ratio $\mathrm{x}=\mathrm{p}_{\|}^{*} / \mathrm{p}_{\max }^{*}$ is the Feynman $\mathrm{x}$-variable, ${ }^{(10)} \mathrm{p}^{*}$ and $\mathrm{E}^{*}$ are the momentum and the energy of the detected particle taken in the center-of-mass system of $\gamma_{v} p\left(p_{\|}^{*}\right.$ is the longitudinal and $p_{1}$ is the transverse component of the momentum with respect to the virtual photon direction and $\mathrm{p}_{\max }^{*}$ is the maximum cms momentum of the detected particle. In general the structure function can depend on the hadron cms energy squared, $s$, and $Q^{2}$.

In Figs. 11-13 we show the integrated structure function

$$
F(x)=\frac{1}{\sigma_{\text {TOT }}} \int \frac{1}{\pi} \frac{\mathrm{E}^{*}}{\mathrm{p}_{\max }^{*}} \frac{\mathrm{d}^{2} \sigma}{\mathrm{dxdp}_{\perp}^{2}} \mathrm{dp}_{\perp}^{2}
$$

normalized to the total cross section at the $W$ and $Q^{2}$ of the data bins shown.

The distributions for the $\mu$-production data include all events (i.e., strange-particle topologies are included). The proton and $\pi^{+}$ambiguity was resolved by ionization up to laboratory momenta of $\sim 1 \mathrm{GeV} / \mathrm{c}$. In addition, 
for those events obtaining a $4 \mathrm{C}$ kinematic fit with a $\chi^{2}<25$ or $1 \mathrm{C}$ with confidence level $>10 \%$, the mass interpretation of the fit was used. For the ambiguous events the proton hypothesis was used for the inclusive proton distributions and the $\pi^{+}$hypothesis was used for the inclusive $\pi^{+}$distribution. Thus, the proton and $\pi^{+}$inclusive distributions for $x$ greater than the values shown in Fig. 11 and 13 represent upper limits on the inclusive distributions. The photoproduction data have been treated in a similar way. The increase of $F(x)$ in the region $\mathrm{x}>0.7$ for $\gamma_{\mathrm{V}} \mathrm{p} \rightarrow \mathrm{p}+$ (anything) may be due to $\pi^{+}$contamination.

Figures $11 b$ and $11 \mathrm{c}$ show $\mathrm{F}(\mathrm{x})$ for slow laboratory protons, i.e., $\mathrm{x} \sim-1.0$. As $Q^{2}$ increases the strong peak at $x \sim-1.0$ disappears. In photoproduction this peak arises from elastic $\rho^{\circ}$ and $\omega$ production.

\section{G. $\mathrm{p}_{\perp}^{2}$ Distributions}

To further study the $Q^{2}$ dependence of the inclusive reaction $\gamma_{\mathrm{v}} \mathrm{p} \rightarrow \mathrm{p}+$ (anything), we discuss next the inclusive variable $\mathrm{p}_{\perp}^{2}$. We limit ourselves to the region of slow protons in the laboratory, $-1 . \leq x \leq-0.5$, a region where the protons are well identified by ionization.

Figure 14 shows $\left\langle\mathrm{p}_{1}^{2}\right\rangle^{-1}$ vs. $\mathrm{Q}^{2}$ for the W range $1.8-4.0 \mathrm{GeV}(<\mathrm{W}>=2.6 \mathrm{GeV})$, where $\left\langle p_{1}^{2}>\right.$ means the mean value of $p_{1}^{2}$ averaged over the experimental distribution; more precisely:

$$
\left\langle\mathrm{p}_{\perp}^{2}\right\rangle=\int_{-1}^{-0.5} \mathrm{dx} \int_{0}^{\infty} \frac{\mathrm{E}^{*}}{\pi \mathrm{p}_{\max }^{*}} \frac{\mathrm{d}^{2} \sigma}{d x \mathrm{dp}_{\perp}^{2}} \mathrm{p}_{\perp}^{2} \mathrm{dp} \mathrm{p}_{\perp}^{2} / \int_{-1}^{-0.5} \mathrm{dx} \int_{0}^{\infty} \frac{\mathrm{E}^{*}}{\pi \mathrm{p}_{\max }^{*}} \frac{\mathrm{d}^{2} \sigma}{d x d p_{\perp}^{2}} \mathrm{dp}_{\perp}^{2}
$$

For an exponential distribution in $\mathrm{p}_{\perp}^{2}$ with slope parameter $\mathrm{B}, \mathrm{B}=\left\langle\mathrm{p}_{\perp}^{2}\right\rangle^{-1}$. The point at $Q^{2}=0$ has been calculated, using the same procedure, from the photoproduction data of reference 4 taken at a fixed energy $\mathrm{W}=2.5 \mathrm{GeV}$ $\left(\mathrm{E}_{\gamma}=2.8 \mathrm{GeV}\right)$. 
We see a tendency for the slope parameter to decrease with $Q^{2}$. This effect is even more striking if we remove the contribution from the diffractive processes $\gamma_{V} p-\left(\rho^{\circ}\right.$ or $\left.\omega^{\circ}\right) p$. For this latter process the slope parameter seems to fluctuate around the photoproduction value, although it is not inconsistent with being flat in $Q^{2} .\left(\chi^{2}\right.$ confidence level $\left.=3 \%\right)$, cf Section IV D.

\section{H. Inclusive $+/-$ Charge Ratio}

Of interest recently has been the inclusive charge ratio $+/-$ as a function of $\mathrm{Q}^{2}$ for $\mathrm{x}$ near 1. ${ }^{(11)}$ In $\mathrm{Fig} .15$ we have plotted the inclusive charge ratio $+/-$ as a function of $Q^{2}$ for four regions of $x$ shown for

$$
2 \leq \mathrm{W} \leq 4 \mathrm{GeV} \text {, or }\langle\mathrm{W}>=2.8 \mathrm{GeV} \text {. }
$$

In the case of proton-pion ambiguities, the pion hypothesis was selected for Fig. 15. Choosing the proton hypothesis gives an $\mathrm{x}$-value more negative than that for the pion because of the mass dependence of the Lorentz transformation to the cms. The photoproduction data were handled in the same way. ${ }^{(9)}$ If we choose the proton hypothesis for the ambiguous events, the $+/$ - ratio for $x>0.3$ decreases by about $20 \%$. We find a significant increase from photoproduction as $Q^{2}$ increases for $\mathrm{x}>0.3$ as observed previously ${ }^{(11)}$ at higher $\mathrm{W}$ values. However, the other $x$ regions show no significant deviation from photoproduction.

\section{CONCLUSIONS}

Due to the preliminary nature of the analysis presented in this paper, all conclusions should be considered with caution, especially when intercomparison of two experiments are involved. This is because the systematic errors in the present analysis are uncertain. 
Given the above cautionary note one can make the following conclusions:

1) In our $Q^{2}$ and $s$ range, for fixed $s$ the charged multiplicity tends to decrease as $Q^{2}$ increases. (Fig. 6)

2) The relative contributions of the $\rho^{\circ}$ and $\omega$ mesons to the total cross sections tend to decrease as $Q^{2}$ increases. (Fig. 7, Fig. 10)

3) The peak at $x \sim-1.0$ in $F(x)$ at $Q^{2}=0$, corresponding to slow protons in the laboratory, decreases rapidly in prominence as $Q^{2}$ increases. (Fig. 11)

4) The $p_{1}^{2}$ distribution for protons with $-1 . \leq x \leq-.5$, and with elastic $\rho$ and $\omega$ removed, flattens by about a factor of 2 in our $Q^{2}$ range as $Q^{2}$ increases. (Fig. 14)

\section{ACKNOWLEDGMENTS}

We wish to acknowledge the significant contribution made by many people at SLAC to this experiment. In particular, the skilled crews of the Bubble Chamber Operations Group under the leadership of Bob Watt persuaded the chamber to run at 10 expansions per second, and thus made the experiment possible. Excellent technical assistance was provided by G. Johnson and W. Weeks. The continuous attentions of the machine operations crews provided us a steady beam at unusually high current and high energy. We wish to thank the scanners in the C.D.A. Group whose skillful measurements resulted in the high efficiency in reducing the data, and $\mathrm{K}$. Eymann and D. Feick for a programming job above and beyond the call of duty. Finally, we thank Dr. W. B. Johnson for invaluable consultation regarding the data processing through TVGP-SQUAW. 


\section{REFERENCES}

1. G. Miller et al., Phys. Rev. D $\underline{5}, 528$ (1972).

2. a) D. O. Caldwell et al., Phys. Rev. Letters 25, 609 (1970).

b) In the review by $H$. W. Kendall in Proceedings of the 1971 International Symposium on Electron and Photon Interactions at High Energy, Cornell, Ed: by N. B. Mistry, Fig. 2 shows values of $\nu \mathrm{W}_{2}$ measured at $4^{\mathrm{O}}$ by SLAC, Group-A.

3. Preliminary results from Group A, LBL.

Private communication from Dr. H. Oberlock, August, 1972.

4. SLAC-LBL-Tufts collaboration, J. Ballam et al. , Phys. Rev. D $\underline{5}, 545$ (1972)

5. SLAC-Weizmann-TelAviv collaboration, Y. Eisenberg et al., Phys. Rev. D $\underline{5}, 15(1972)$.

6. P. Söding, Phys. Letters 19, 702 (1965).

7. K. Schilling et al., Nuc. Phys. B15, 397 (1970).

R. Thews, Phys. Rev. 175,1749 (1968).

8. Aachen-Berlin-Bonn-Hamburg-München Collaboration, Phys. Rev. 175, 1669 (1968),

J. Ballam et al. , Phys. Rev. Letters $\underline{24}, 1364$ (1970); 26, 155 (E).

"Vector Meson Production by Polarized Photons at 2.8, 4.7, and 9.3 GeV/c",

J. Ballam et al. , SLAC-LBL-TUFTS, SLAC-PUB-1143 (1970).

9. The photoproduction data of the SLAC-LBL-Tufts collaboration was analyzed in the same way as the inelastic $\mu-\mathrm{p}$ data, and is described in K. Moffeit et al. , Phys. Rev. D $\underline{5}, 1603$ (1972), for the $\pi^{-}+($anything) case The $\pi^{+}$and proton inclusive distributions shown in the figures will be described more fully in a following publication. 
A correction of $1.3 \%$ at $2.8 \mathrm{GeV}$ and $2.9 \%$ at $4.7 \mathrm{GeV}$ has been applied to the normalized distributions given in this paper to account for the $\pi$ particles in the strange particle topologics. For $\gamma p \cdots \pi^{+-}+$(anything) the 1-prongs were obtained from a scan of about $10 \%$ of the film. Since about $14 \%$ of these 1-prongs come from the low energy tail of a few percent extending towards low energies, the $\pi^{+}$distribution for $p_{1 a b}<500 \mathrm{MeV}$ may be contaminated. Subtraction of all 1-prongs reduces the stricture function by about $50 \%$, at $x=-1$ and by about $10 \%$ at $x=-0.5$. For $\gamma p \rightarrow p+($ anything) the $1-$ prong events were excluded as were the strange particle topologies. The $\mathrm{x}$ distributions were normalized to the total cross section remaining after these subtractions. We point out that the proton distributions from these events may be different than those of the topologies shown.

The elastic $\rho^{o}$ and $\omega$ contributions have been removed by the simplest cuts: All events of $\gamma \mathrm{p} \rightarrow \pi^{+} \pi^{-} \mathrm{p}$ with $\mathrm{M}_{\pi^{+}}^{+} \pi^{-}<1.0 \mathrm{GeV}$ are referred to as "elastic" $\rho^{\circ}$ events and all events of $\gamma \mathrm{p} \rightarrow \pi^{+} \pi^{-} \pi^{\circ} \mathrm{p}$ with $\left|\mathrm{M} \pi^{+} \pi^{-} \pi^{-\mathrm{O}^{-}}\right|<20 \mathrm{MeV}$ are referred to as "elastic" $\omega$ events. In addition, the calculated photon energy was required to be within the energy peak of the photon spectrum for the $\omega$ events.

10. R. P. Feynman, Phys. Rev. Letters 23, 1415 (1969).

11. J. T. Dakin et al., Phys. Rev. Letters $\underline{29}, 746$ (1972).

12. For a summary of these measurements see the review by K. Berkelman in Proceedings of the 1971 International Symposium on Electron and Photon Interactions at High Energies, Cornell, Ed. by N. B. Mistry.

13. Peter Joos, "Compilation of Photoproduction Data Above $1.2 \mathrm{GeV}$," DESY-HERA 70-1 (1970). 
14. P. Eberhard and M. Pripstein, Phys. Rev. Letters 10, 351 (1963). Here we discarded those events with $\mathrm{M}_{\pi^{+} \mathrm{p}}<1.325 \mathrm{GeV}$ and used those with $\mathrm{M}$ - $<1.325 \mathrm{GeV}$ twice. $\pi \mathrm{p}$

15. T. Carroll and M. Della Negra, Internal Note, Group-B.C. (BC Note 16) Stanford Linear Accelerator Center. 


\section{FIGURE CAPTIONS}

1. The hatched region shows the trend of the measured values of the total photoabsorption cross section $\sigma_{\mathrm{T}}+\sigma_{\mathrm{S}}$ on hydrogen for $\mathrm{W}=3$ to $5 \mathrm{CeV}$. The values for real photons $\left(Q^{2}=0\right)$ are from ref $2 a$. The values for virtual photons are derived from data given in ref. $2 \mathrm{~b}$; since $\epsilon$ is near unity and $\sigma_{\mathrm{T}} \approx .2 \sigma_{\mathrm{S}}$, no significant uncertainty is introduced in the extrapolation to $\epsilon=1$.

2. A schematic representation of the experiment from the origin of the muon beam to the muon telescope behind the bubble chamber. The upper part of the figure, depicting the muon beam, is not to scale.

3. A photograph of a typical event showing the full beam of approximately $100 \mu$-tracks in the bubble chamber. The inelastic interaction can be seen near the right edge of the beam. The $\mu$ scatters forward and to the left, and 5 charged hadrons can be seen emerging from the vertex.

4. $(a, b)$ This shows the distribution of our data in a $W-Q^{2}$ plane. In Fig. 4a we show superimposed on the data contours of our detection probability $P$. These values are calculated by a Monte Carlo technique. Below in Fig. 4b, we show again the same data but superimpose contours of $\epsilon$, the virtual photon longitudinal to transverse polarization ratio, and $\omega^{\prime}\left(=1+\mathrm{W}^{2} / \mathrm{Q}^{2}\right)$, the scaling variable.

5. $(\mathrm{a}, \mathrm{b}, \mathrm{c})$ Total cross section values $\sigma_{\mathrm{T}}+\epsilon \sigma_{\mathrm{S}}$ obtained from the data by extracting the virtual photon flux factor from the measured cross sections. The polarization parameter $\epsilon$ is the ratio of longitudinal to transverse polarization of the virtual photons, and varies from .8 to 1.0 in these data. No attempt has been made to separate the longitudinal and transverse parts of the cross section. The solid line is the single arm electroproduction 
values for $\sigma_{T}+\epsilon \sigma_{S}$ averaged over the same $W$-values as the data. Beneath the total cross sections, each point is broken into fractional contributions from different topoligies. Only outgoing charged hadrons are counted as prongs. The photoproduction points shown are from ref 3 . for the $\mathrm{W}=1.4-1.8 \mathrm{GeV}$ data, and from ref. 4 for the $\mathrm{W}=1.8-2.8$ and 2.8-3.8 GeV data.

5. (d) 1C Fits: This shows further breakdown of the 1-prong contribution to the total cross section for the values $W=1.8-2.8 \mathrm{GeV}$. For the 1-prong topology, the $1 \mathrm{C}$ hypotheses are $\gamma_{\mathrm{v}} \mathrm{p} \rightarrow \mathrm{p} \pi^{\mathrm{o}}$ and $\gamma_{\mathrm{v}} \mathrm{p} \rightarrow \pi^{+} \mathrm{n}$. One C fits are rejected if the probability for the hypothesis is less than $4 \%$. Corrections have been applied for events falling below this cut. In many cases (typically 50\%), where one hypothesis works, the other is also an acceptable fit. The photoproduction points shown come from data of ref. 4 and ref. 13 .

6. (a) The charged hadronic multiplicity as a function of $Q^{2}$. The $Q^{2}$ intervals used were $.15 \leq \mathrm{Q}^{2} \leq 0.4(\mathrm{GeV} / \mathrm{c})^{2}, 0.4 \leq \mathrm{Q}^{2} \leq 0.8(\mathrm{GeV} / \mathrm{c})^{2}$, $0.8 \leq Q^{2} \leq 2.5(\mathrm{GeV} / \mathrm{c})^{2}$. Three $W$ regions are shown: Bottom, $\langle W\rangle=1.6 \mathrm{GeV}$ for the range $1.4 \leq \mathrm{W} \leq 1.8 \mathrm{GeV} ;$ Middle, $\langle\mathrm{W}>=2.25$ for the range $1.8 \leq \mathrm{W} \leq 2.8 \mathrm{GeV}$; Top, $\langle\mathrm{W}\rangle=3.25 \mathrm{GeV}$, for the range $2.8 \leq \mathrm{W} \leq 3.8 \mathrm{GeV}$. The photoproduction points were obtained from ref. 3 and ref. 4.

6. (b) Average numbers of charged hadronic tracks in three regions of $\mathrm{x}$ as a function of $\mathrm{Q}^{2}$ for the $\mathrm{W}$ range $2-4 \mathrm{GeV}$. The photoproduction values $\left(Q^{2}=0\right)$ were obtained from the $W=2.5 \mathrm{GeV}$ data, ${ }^{(4)}$ and scaled up so that $\langle\mathrm{N}\rangle$ summed over all $\mathrm{x}$ equalled the photoproduction multiplicity interpolated to $\langle\mathrm{W}\rangle=2.85 \mathrm{GeV}$. 
7. (a) The dipion mass spectrum from which the ratios below were derived. The superimposed curve is taken from ref. 4 and represents the spectrum observed in $\mathrm{W}=2.8 \mathrm{GeV}$ photoproduction.

7. (b) Cross sections for observed $\rho^{0} \mathrm{~s}$ in the reaction $\mu^{-} \mathrm{p} \rightarrow \mu^{-} \mathrm{p} \rho^{0}$ for W between 2 and $4 \mathrm{GeV}$, normalized to the total hadronic cross section. The triangle represents the photoproduction value averaged over our photon spectrum for the same interval in $\mathrm{W}$.

8. Dependence of $\rho^{\circ} \mu$-production on the 4-momentum transfer to the proton, $t$, for two intervals of $Q^{2}$ and $2.0<W<4.0 \mathrm{GeV}$. No correction for $t_{\min }$ has been applied. The dotted line indicates the dependence found for $\mathrm{W}=2.8 \mathrm{GeV} \rho^{\circ}$ photoproduction $\left(\mathrm{B}=5.6 \mathrm{GeV}^{-2}\right)$.

9. The angular distribution for $\rho^{\mathrm{O}}$ decay in the helicity system. For $\cos \theta \approx-1$ there is a peak corresponding to a small contamination by $\Delta^{++} \pi^{-}$production. The $\cos ^{2} \Psi$ dependence comes from transversely polarized photons only and so the non zero values at $\Psi=90^{\circ}$ and $270^{\circ}$ may be due to longitudinal photons.

10. (a) $\mathrm{M}\left(\pi^{+} \pi^{-} \pi^{\mathrm{o}}\right)$ from fits to $\mu^{-} \mathrm{p} \rightarrow \mu^{-} \mathrm{p} \pi^{+} \pi^{-} \pi^{\circ}$ with $\mathrm{Q}^{2} \geq .15(\mathrm{GeV} / \mathrm{c})^{2}$. For 1C fits we plot fits with a confidence level greater than $10 \%$. The shaded events have $Q^{2} \geq .5(\mathrm{GeV} / \mathrm{c})^{2}$. The distribution is not weighted by our acceptance and there is no cut on $\mathrm{W}$.

10. (b) Ratio of the cross section $\sigma\left(\gamma_{\mathrm{v}} \mathrm{p} \rightarrow \mathrm{p} \omega\right) / \sigma_{\text {TOT }}$ for $1.8<\mathrm{W}<3.9 \mathrm{GeV}$ plotted vs. $Q^{2}$. For comparison we plot the photoproduction point at $Q^{2}=0$. 
11. (a) Reaction $\gamma_{\mathrm{v}} \mathrm{p} \rightarrow \mathrm{p}+$ (anything): Normalized structure function $F(x)$ vs. $x$ for the indicated $W$ and $Q^{2}$ intervals. To the right of the symbol $/$ some $\pi^{+}$contamination occurs. Photoproduction data at $Q^{2}=0$ are taken from ref. 4. The dotted curves are approximations to the photoproduction data for comparison to the $Q^{2}=0$ distributions. Elastic $\rho^{\circ}$ and $\omega$ events have been excluded from some points as noted.

11. (b, c) Reaction $\gamma_{\mathrm{v}} \mathrm{p} \rightarrow \mathrm{p}+$ (anything): Normalized structure function $\mathrm{F}(\mathrm{x})$ vs. $\mathrm{x}$ for the indicated $\mathrm{Q}^{2}$ intervals for (b) $1.8<\mathrm{W}<2.5 \mathrm{GeV}$, (c) $2.5<\mathrm{W}<4.0 \mathrm{GeV}$. The photoproduction data at $\mathrm{Q}^{2}=0$ are taken from ref. 4. Elastic $\rho^{\circ}$ and $\omega$ events have been excluded from some points as noted.

12. Reaction $\gamma_{v} p \rightarrow \pi^{-}+($anything): Normalized structure function $F(x)$ vs. $x$ for the indicated $W$ and $Q^{2}$ intervals. Photoproduction data at $Q^{2}=0$ are taken from ref. 4. Dotted curves are approximations to the photoproduction data for comparison to the $\mathrm{Q}^{2}=0$ distributions. Elastic $\rho^{0}$ and $\omega$ events have been excluded from some points as noted.

13. Reaction $\gamma_{\mathrm{v}} \mathrm{p} \rightarrow \pi^{+}+$(anything): Normalized structure function $F(x)$ vs. $x$ for the indicated $W$ and $Q^{2}$ intervals. To the right of the symbol $\&$ some proton contamination occurs. Photoproduction data at $Q^{2}=0$ is taken from ref. 4. Dotted curves are approximations to the photoproduction data for comparison to the $\mathrm{Q}^{2}=0$ distributions. Elastic $\rho^{\circ}$ and $\omega$ events have been excluded from some points as noted. 14. $\left\langle\mathrm{p}_{\perp}^{2}\right\rangle^{-1}$ as a function of $\mathrm{Q}^{2}$ for backward protons in the inclusive reaction $\gamma_{\mathrm{v}} \mathrm{p} \rightarrow \mathrm{p}+$ (anything) for $-1 \leq \mathrm{x} \leq-0.5$ in the W range $1.8-4 \mathrm{GeV}$. The upper graph is for all events. The middle graph is for events with a $4 \mathrm{C}$ elastic $\rho^{\circ}$ or $\omega$. The lower graph is for all events that do not have an elastic $\rho^{\circ}$ or $\omega$. 
15. The inclusive charge ratio $(+/-)$ as a function of $Q^{2}$ for various $x$ ranges. In the case of proton-pion ambiguities, the pion hypothesis was selected. The photoproduction points were obtained from ref. 4. Also shown in the region $x>0.3$ are the lowest two $Q^{2}$ points of ref. 11 for which $\langle W\rangle$ was about $4 \mathrm{GeV}$. 


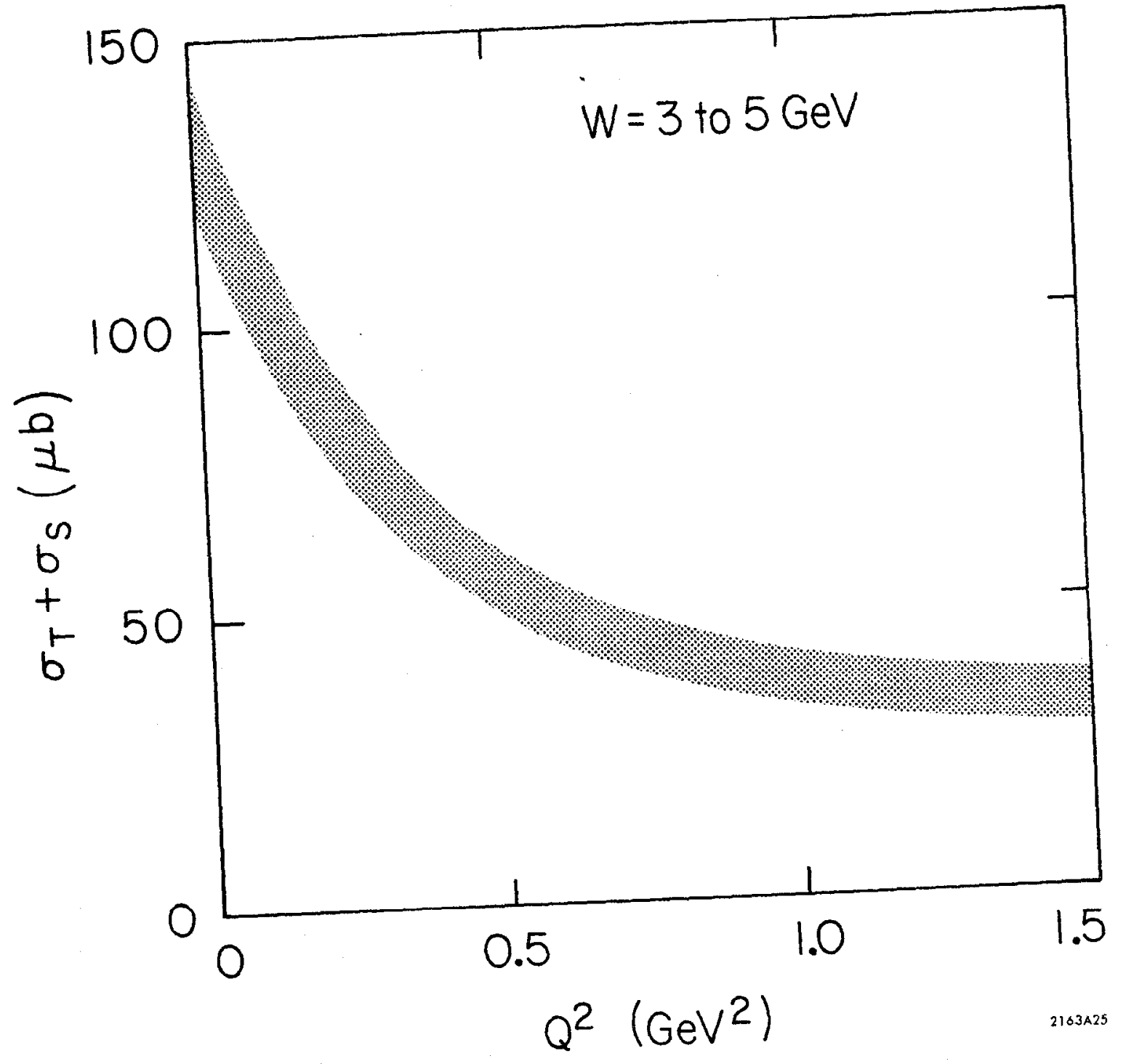

Fig. I 

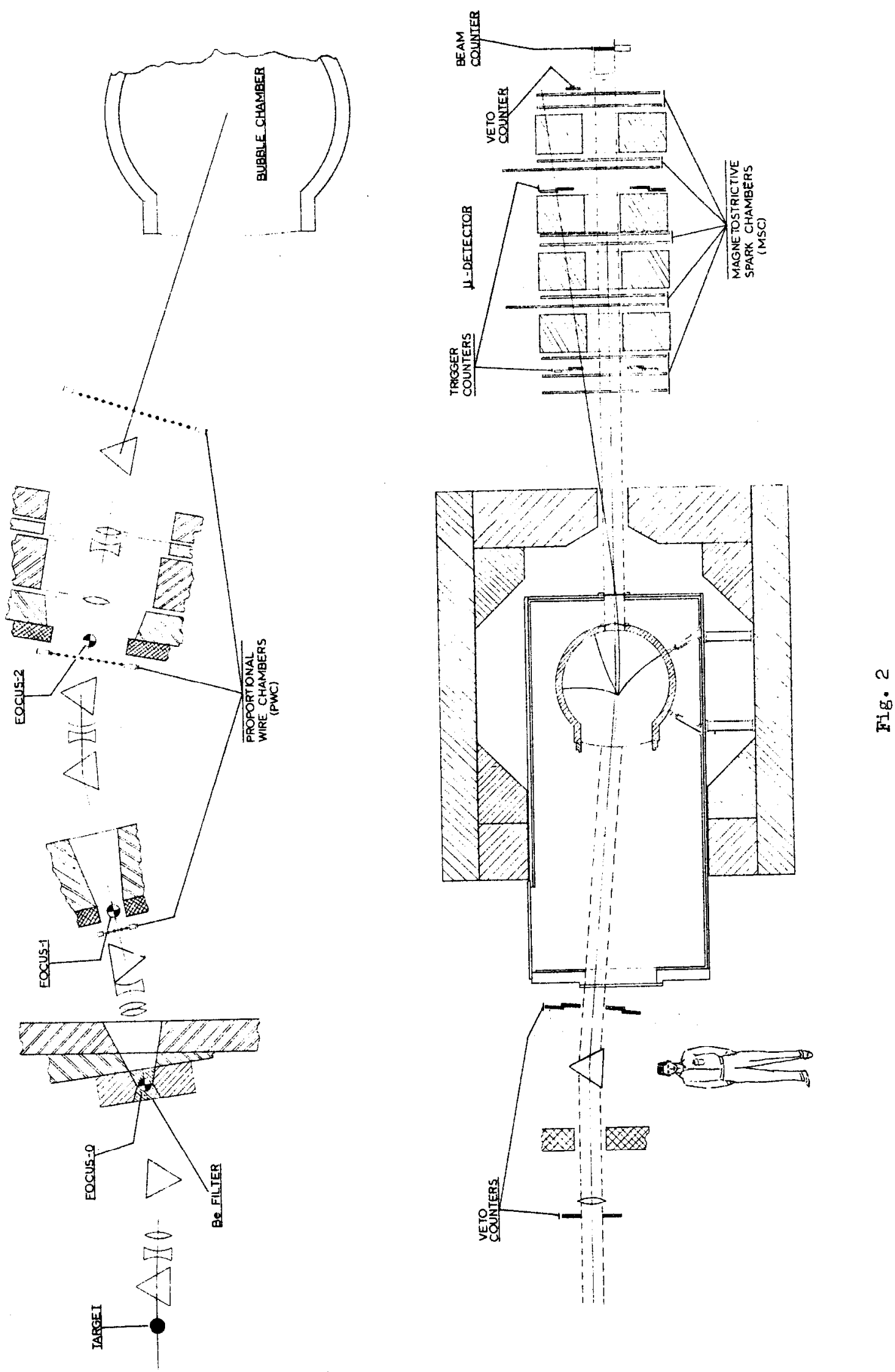


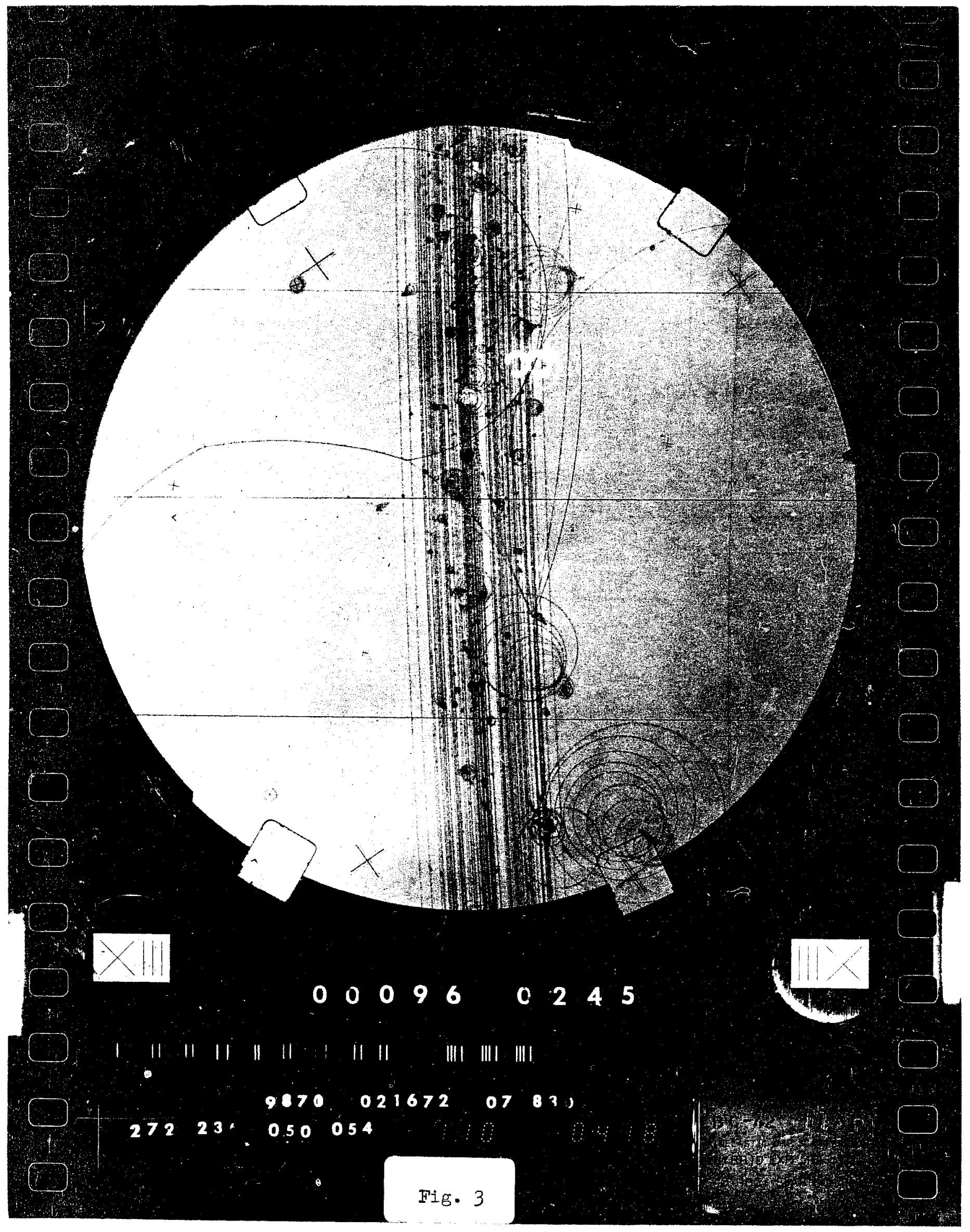




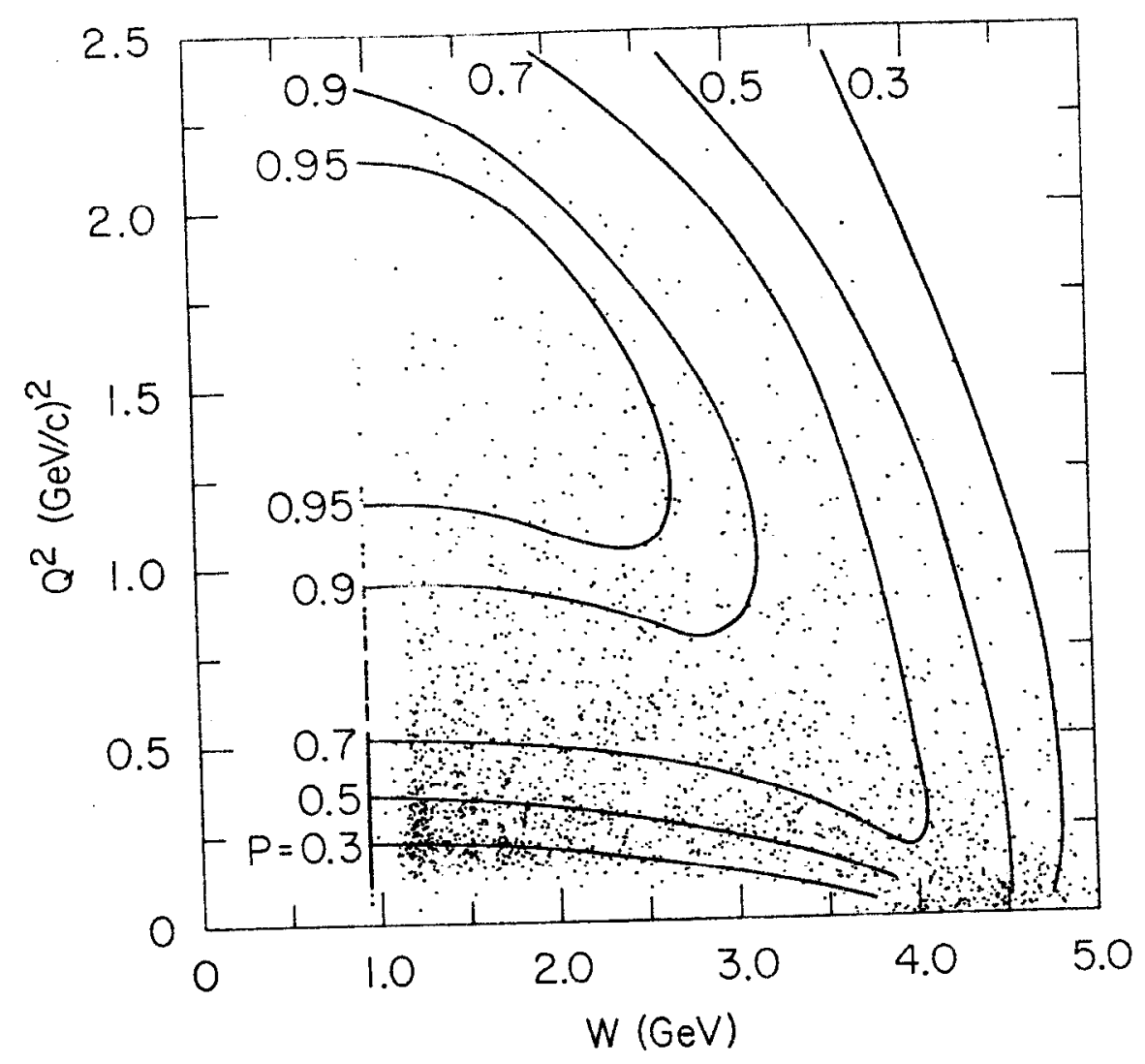

Fig. $4 a$

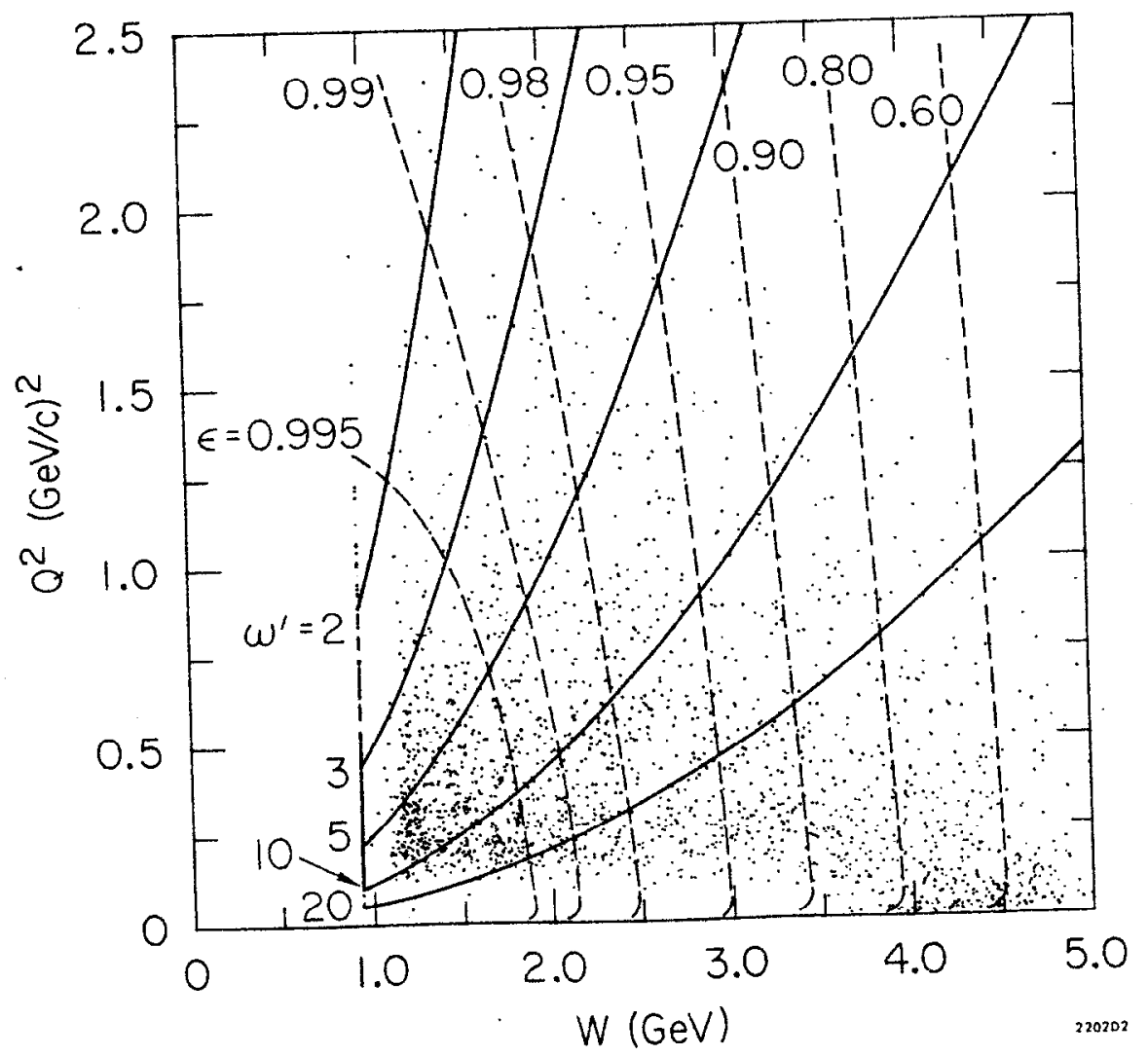

Fig. $4 b$ 


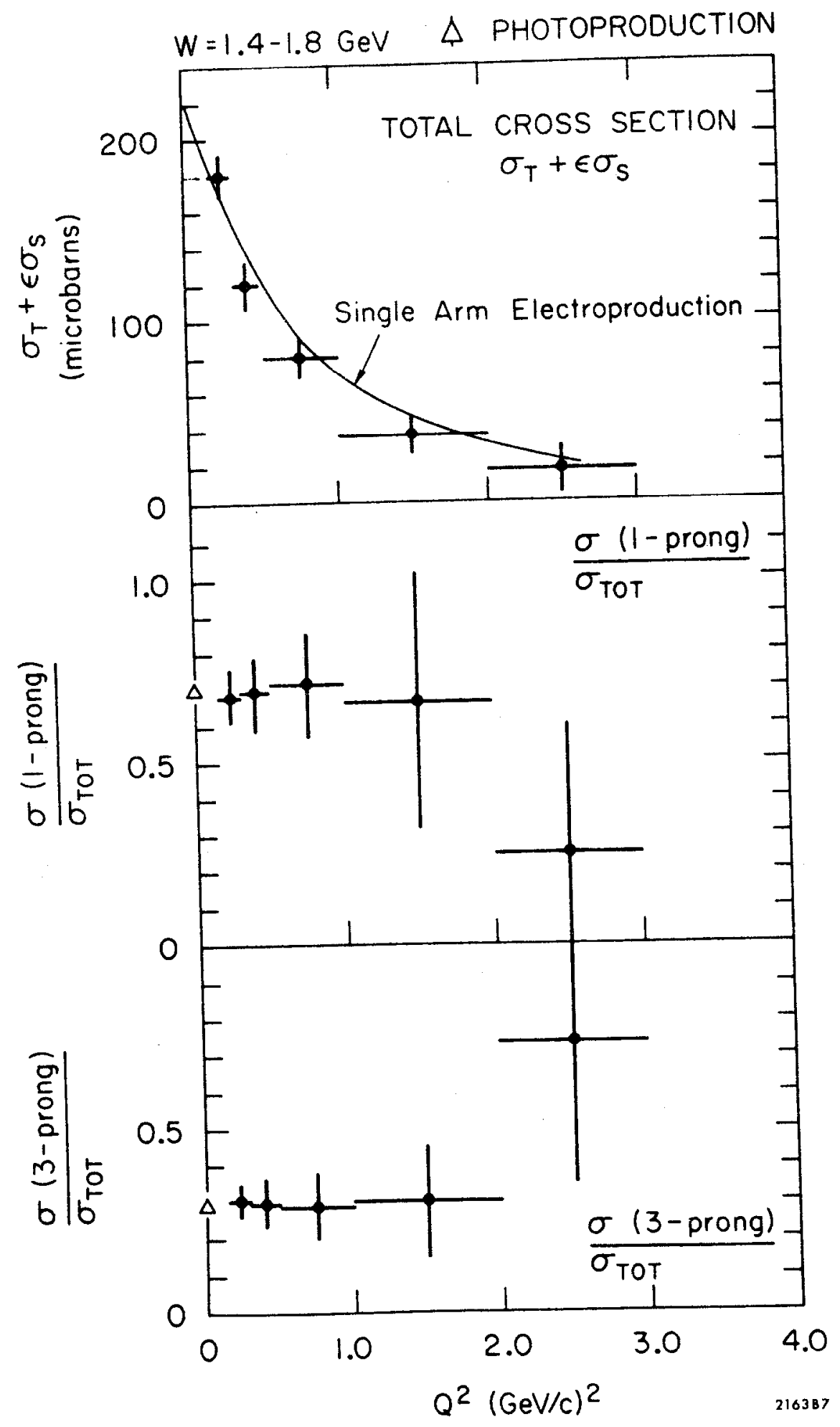

Fig. 5 a 


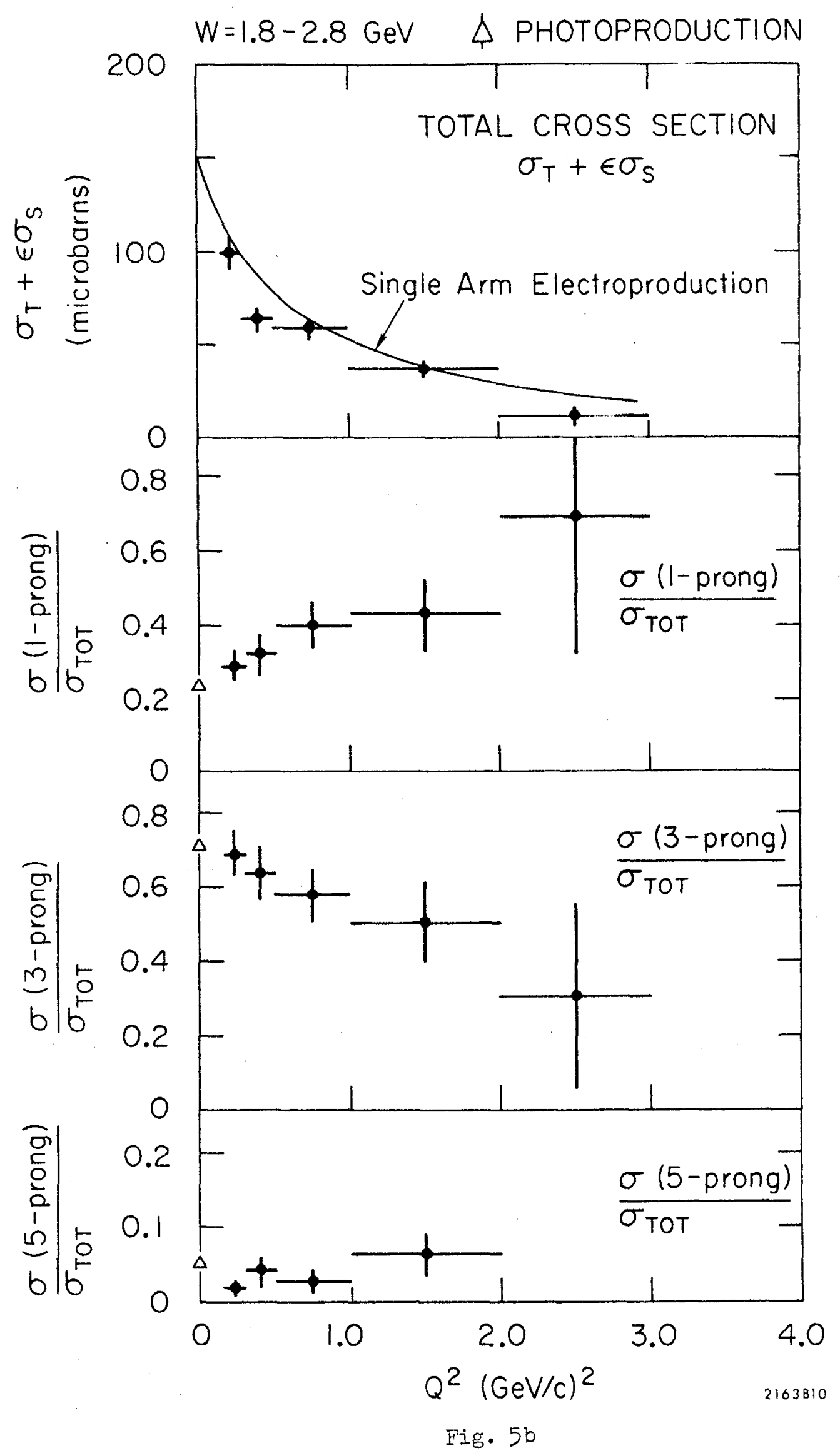




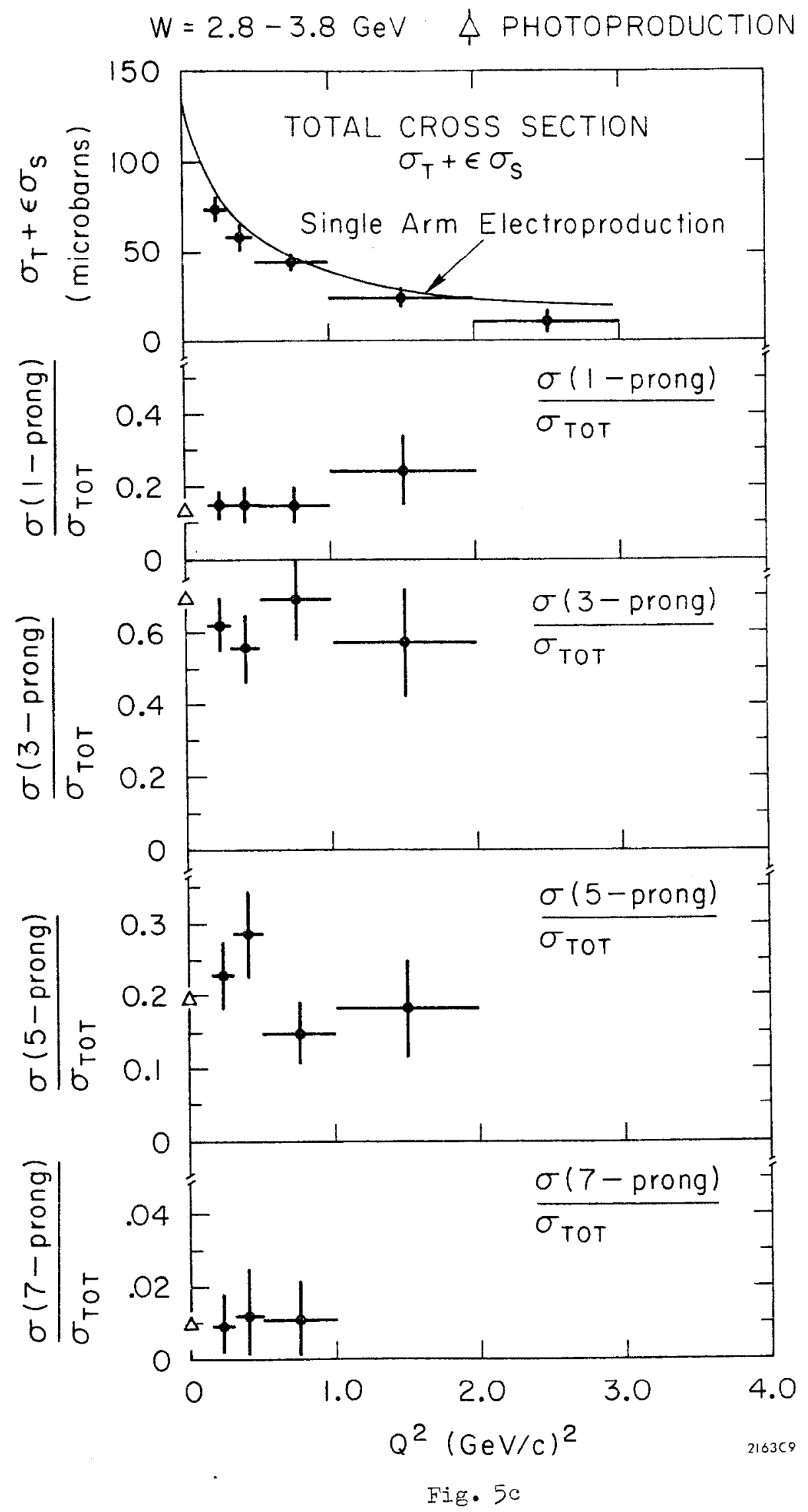




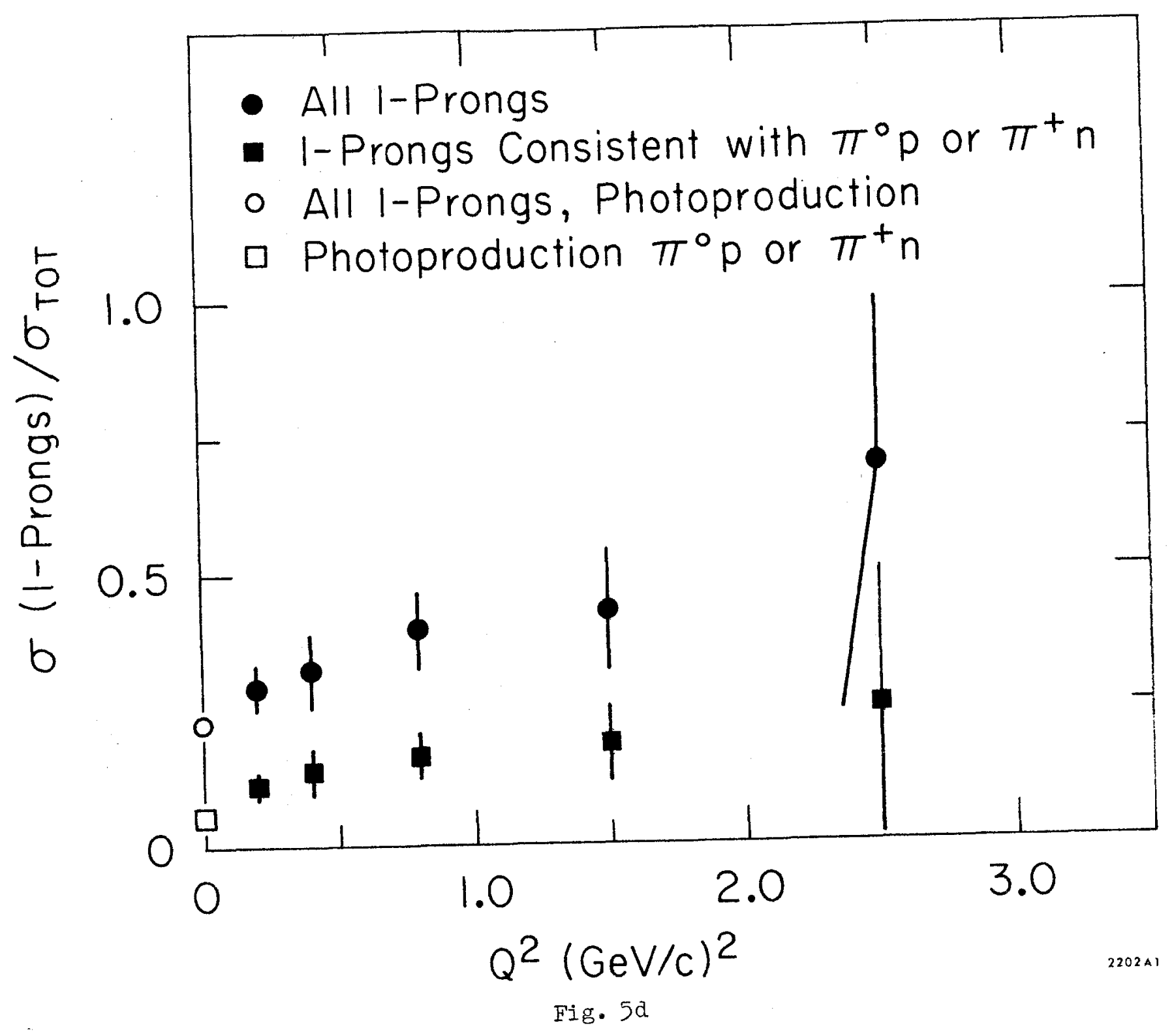




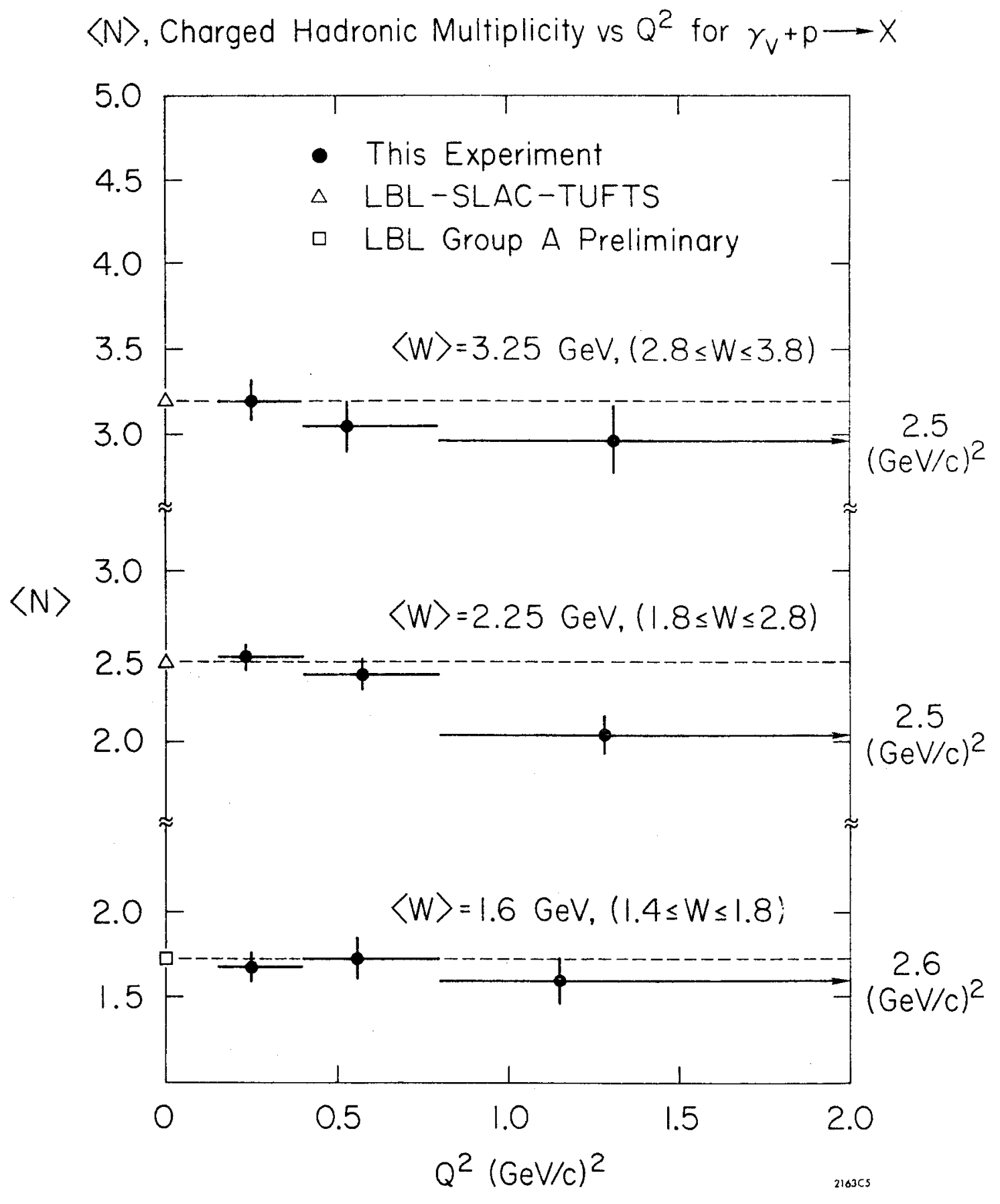

Fig. 6a 


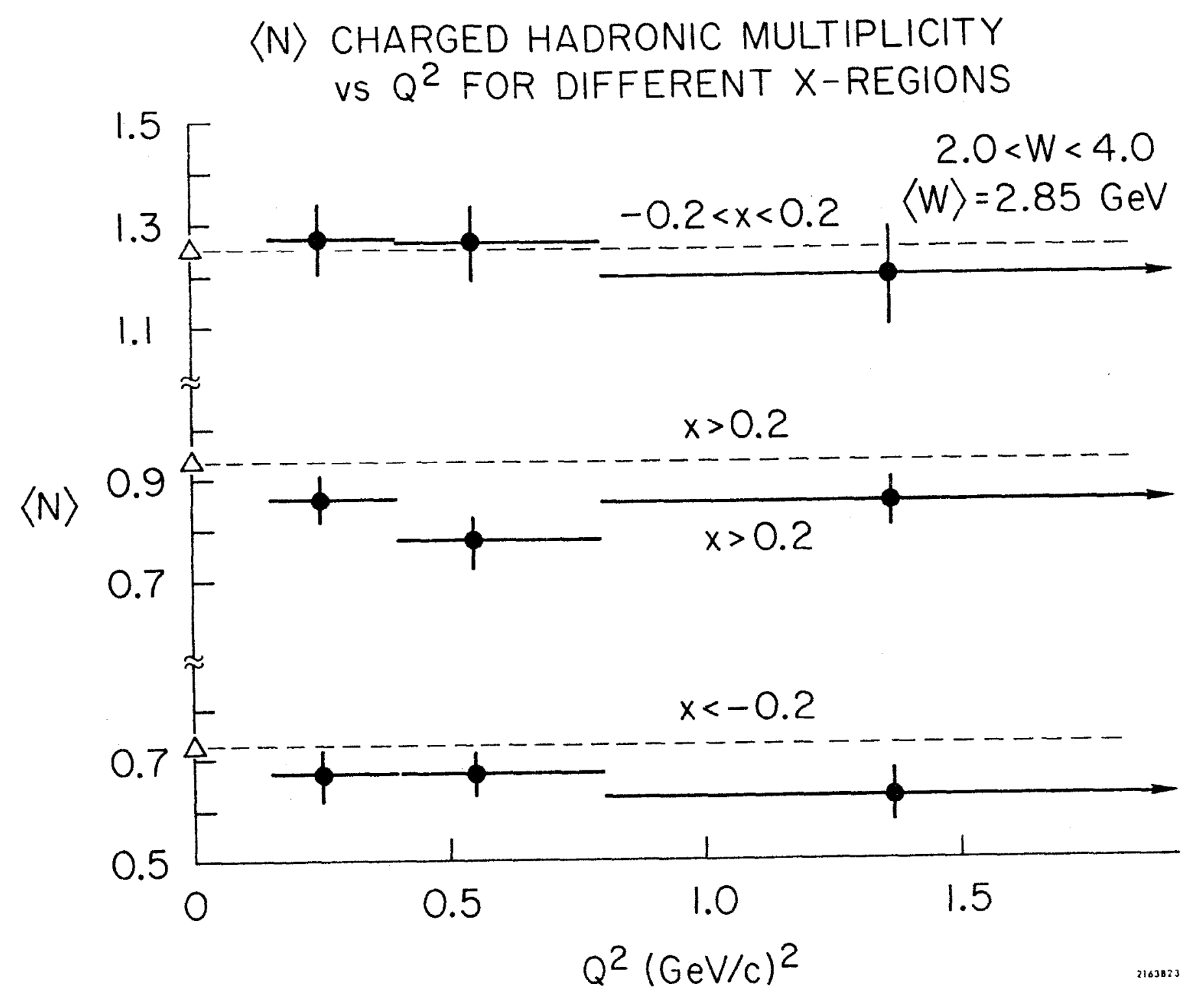

Fig. $6 \mathrm{~b}$ 


$$
\gamma_{v} P \rightarrow \rho^{\circ} \mathrm{P}
$$
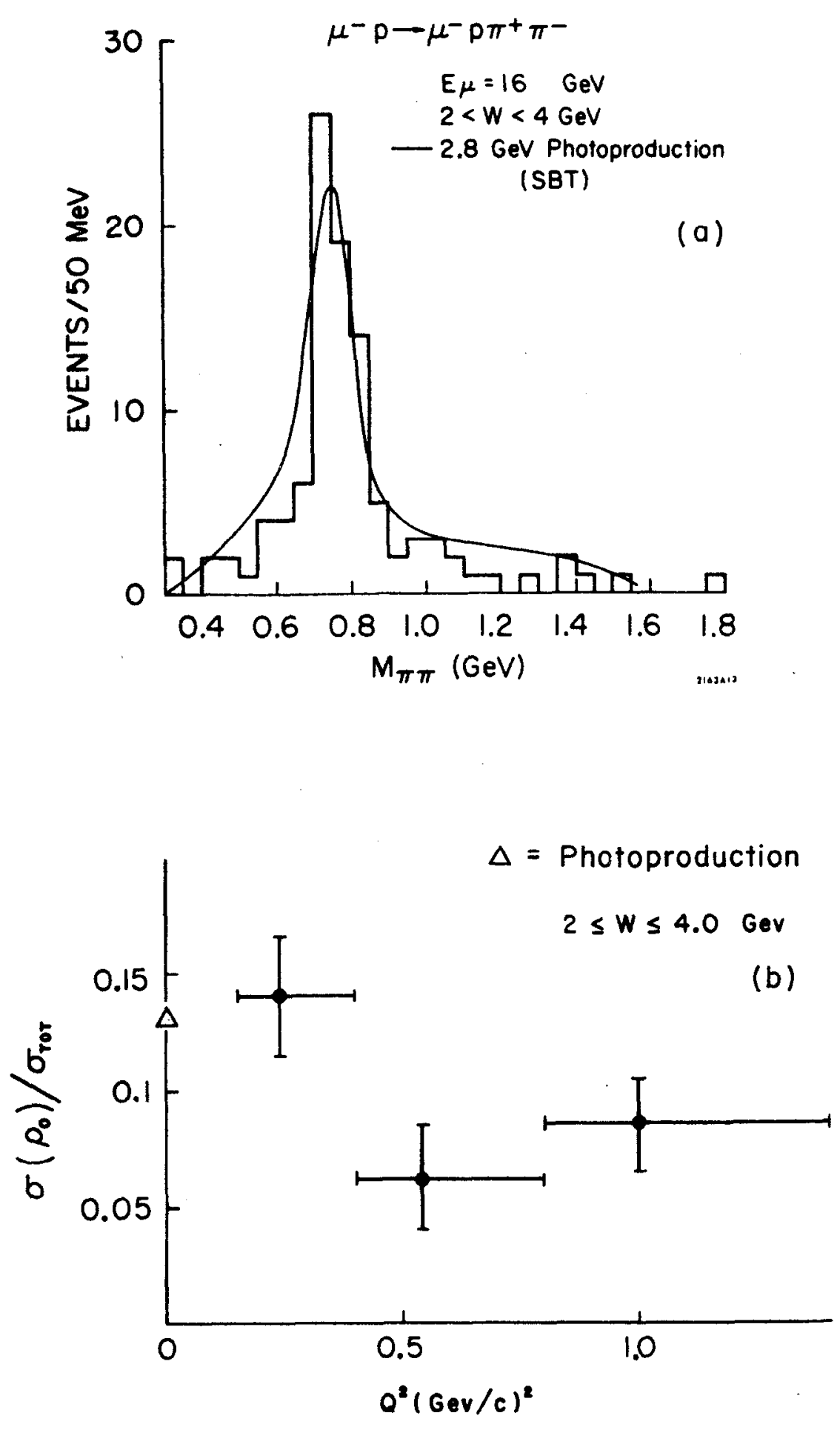

Fig. 7 


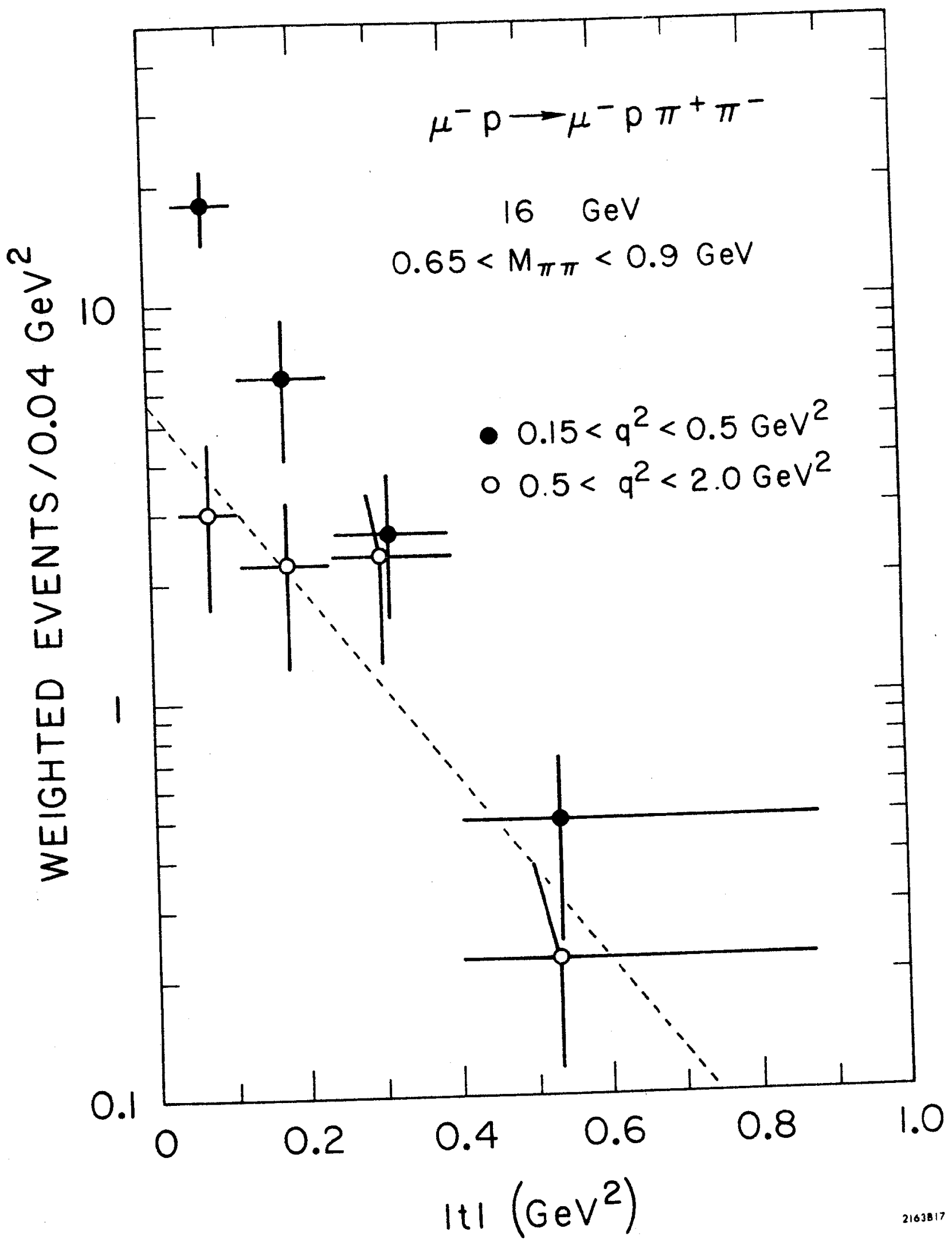

Fig. 8 

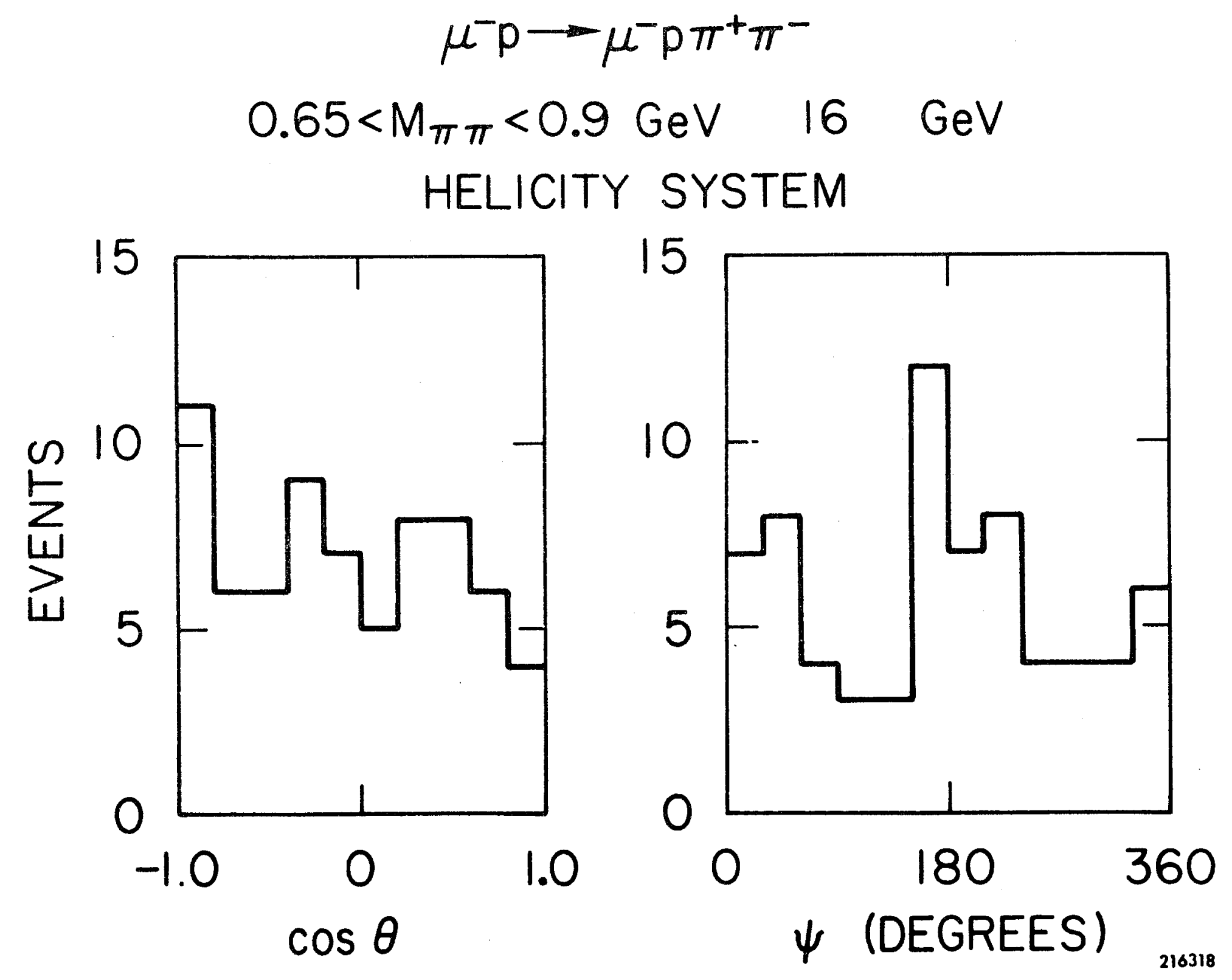

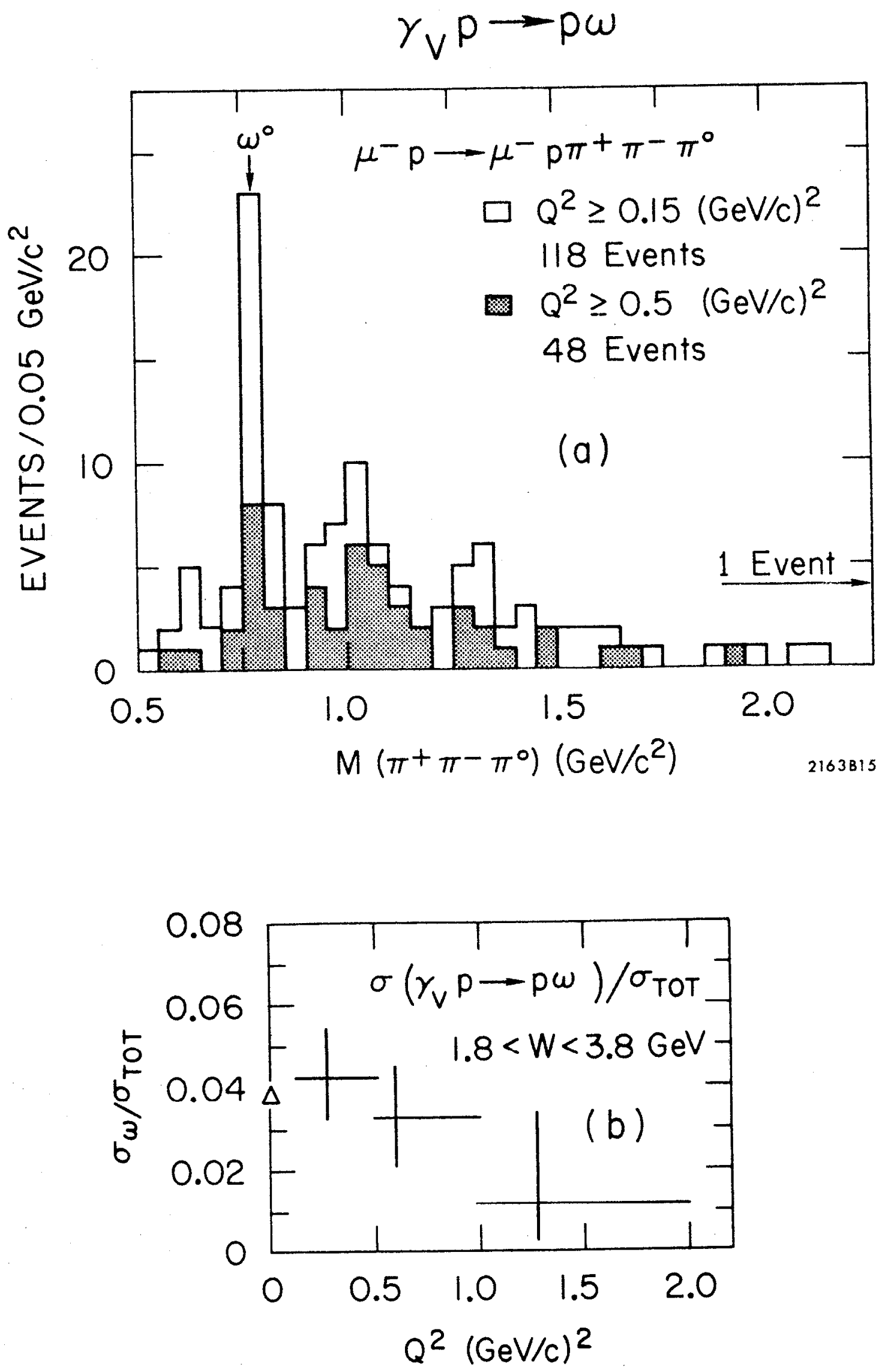

Fig. 10 


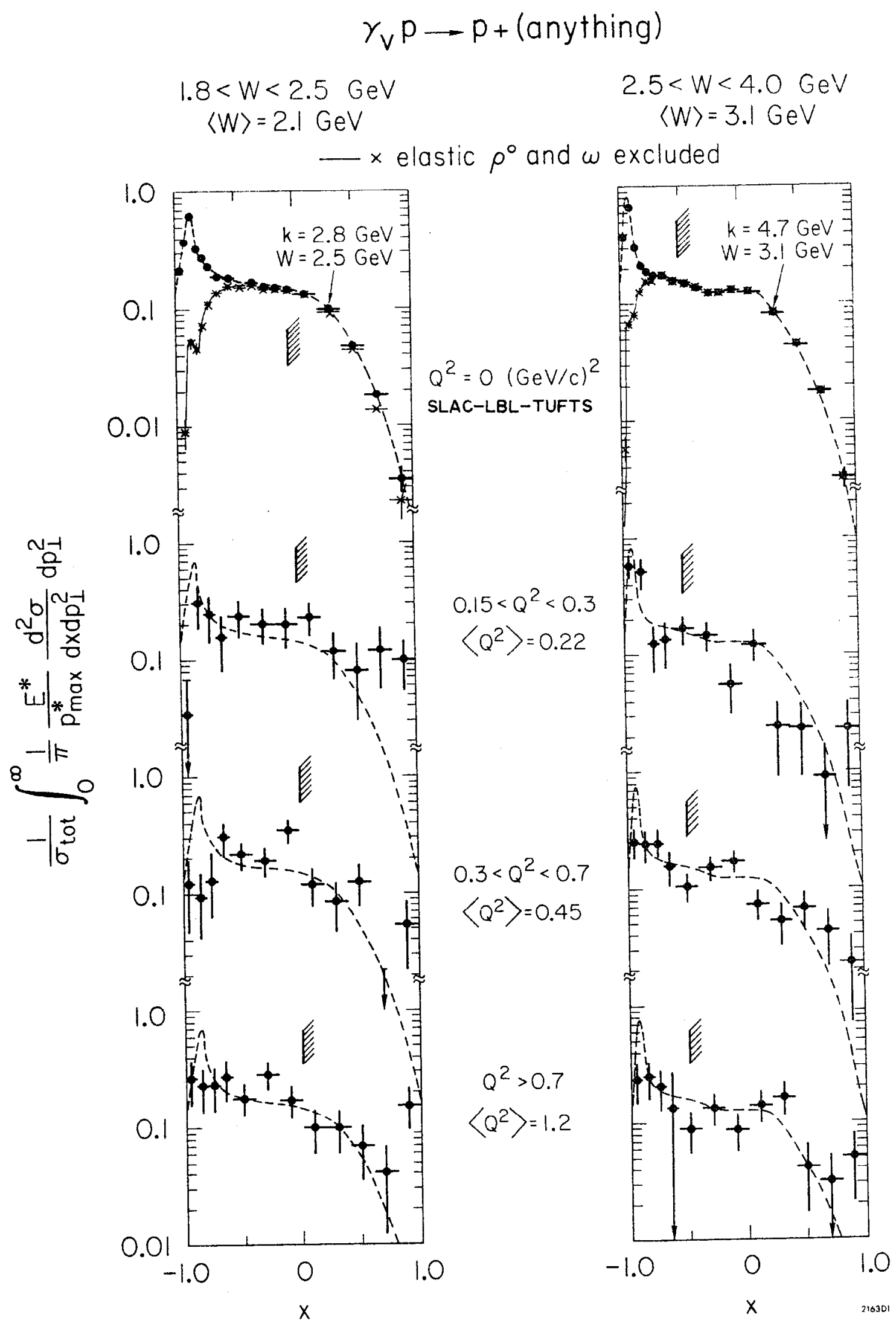

Fig. ila 


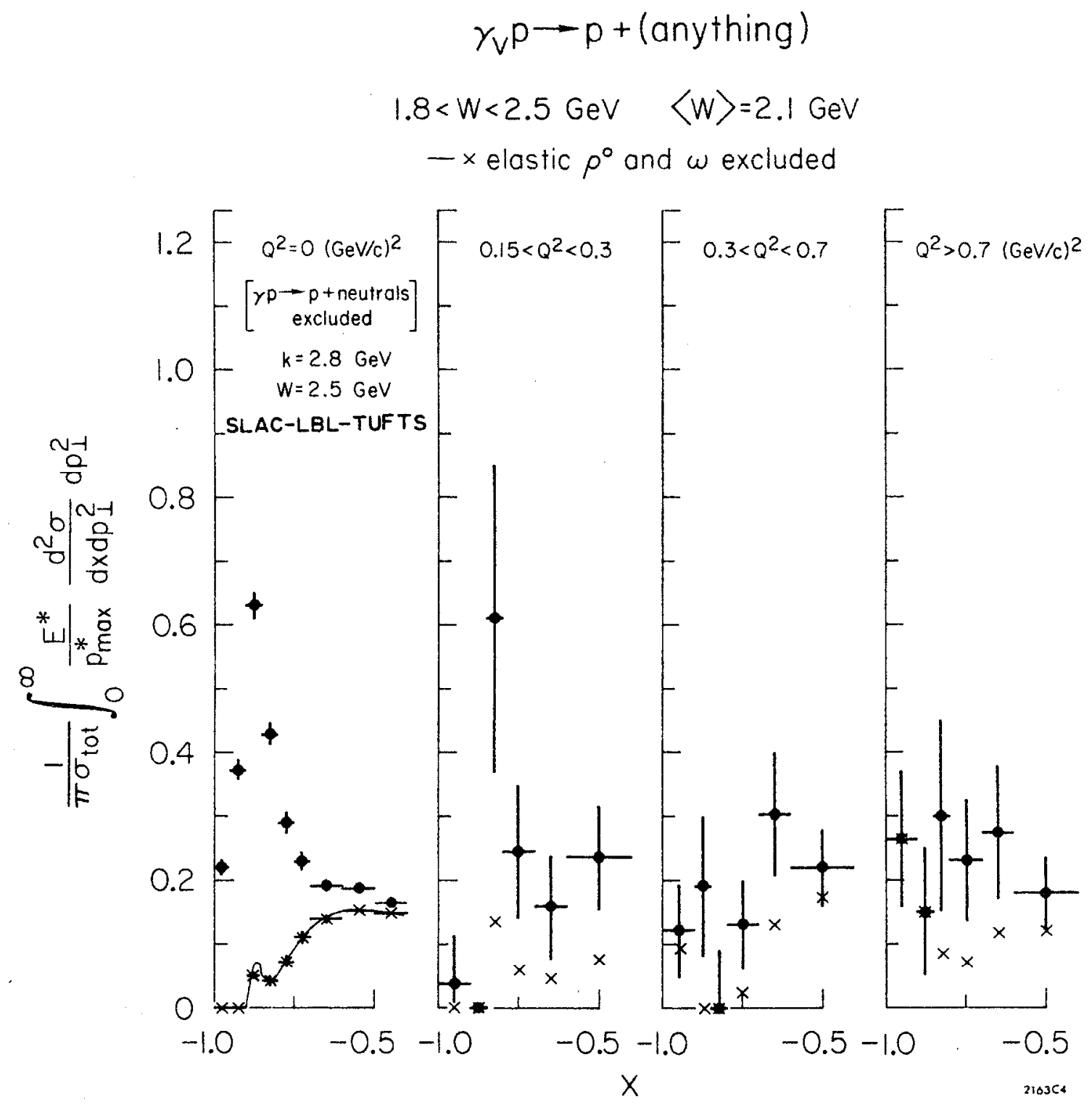

Fig. IIb 


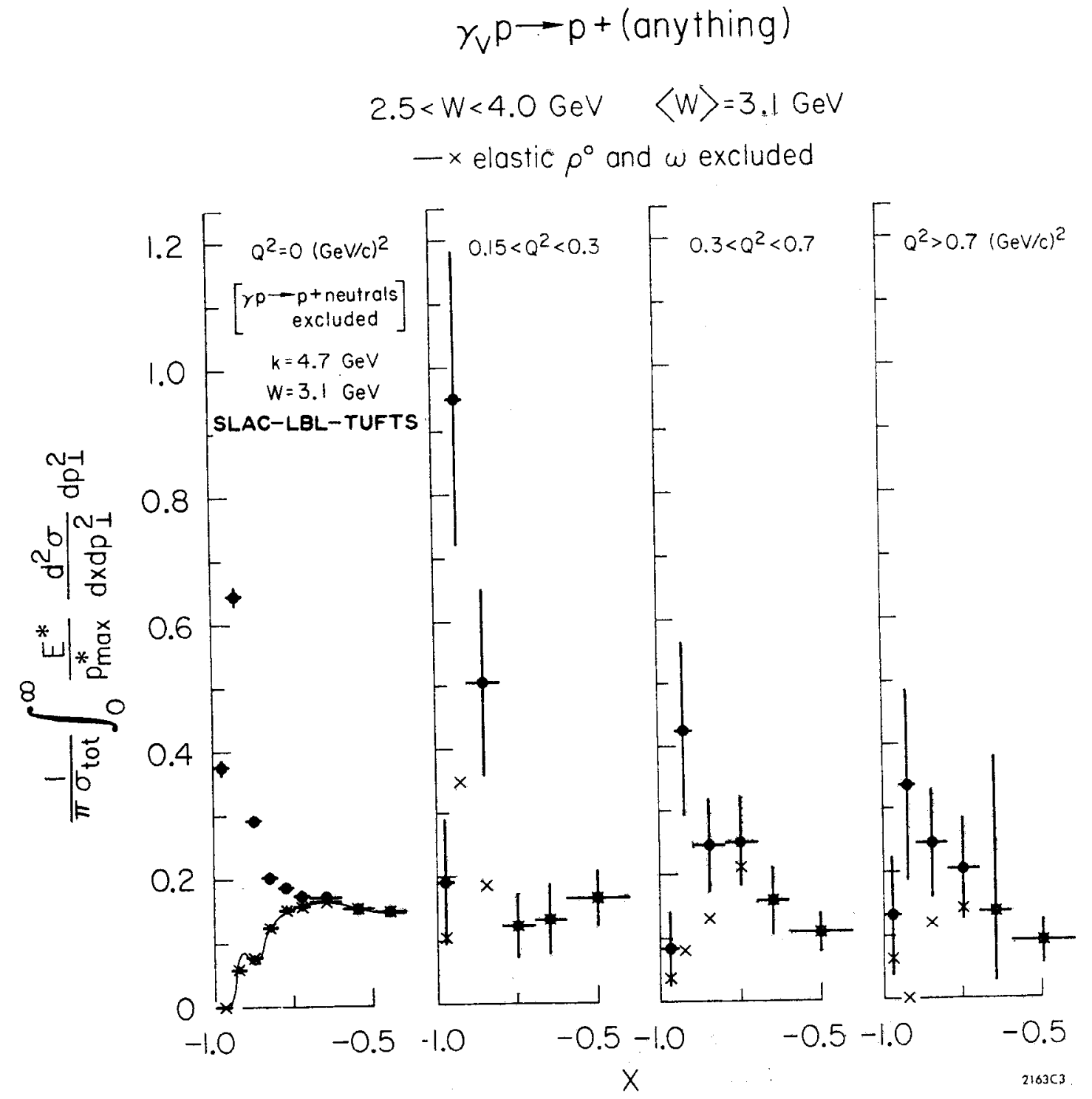

Fig. Ilc 


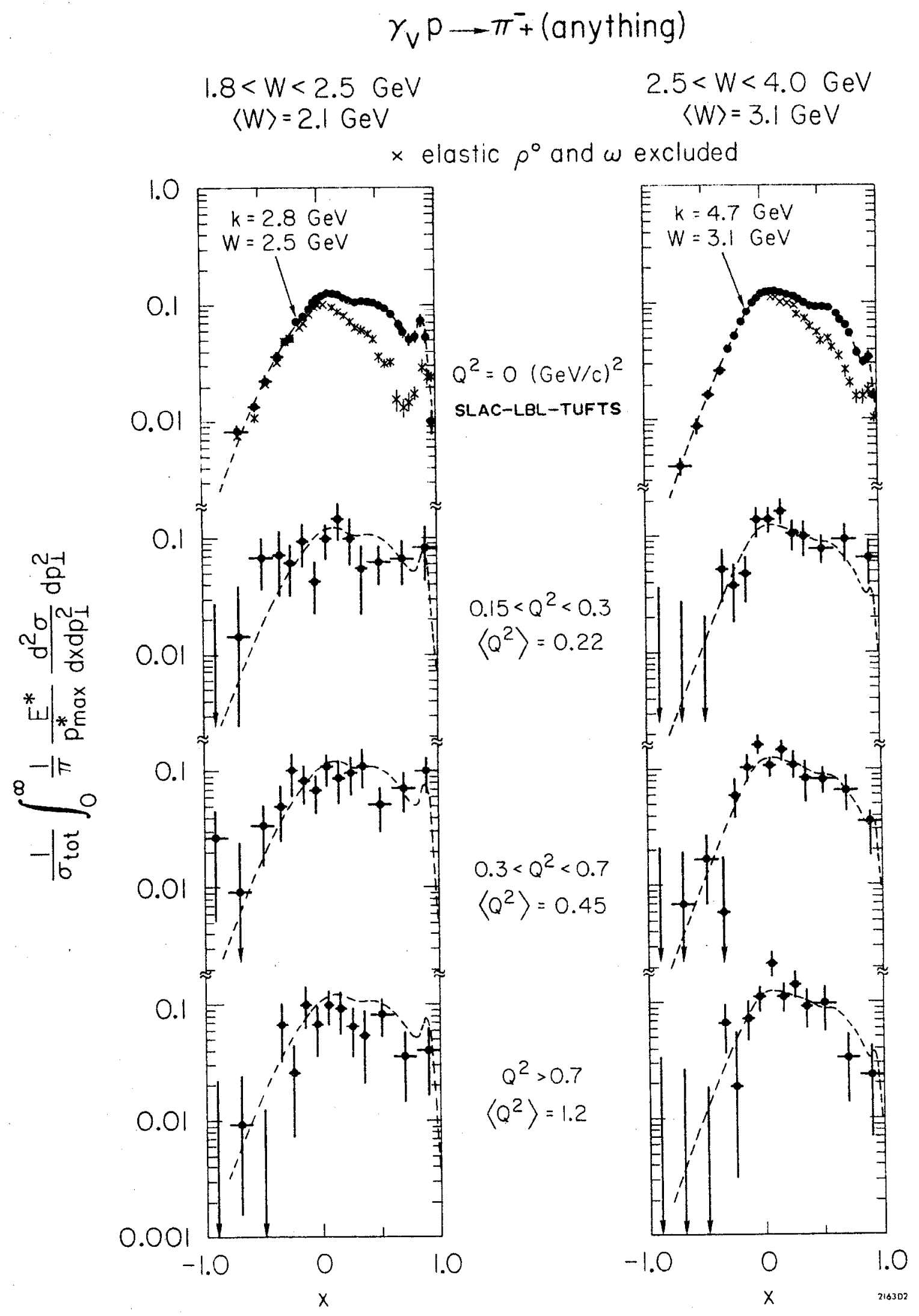

Fig. 12 


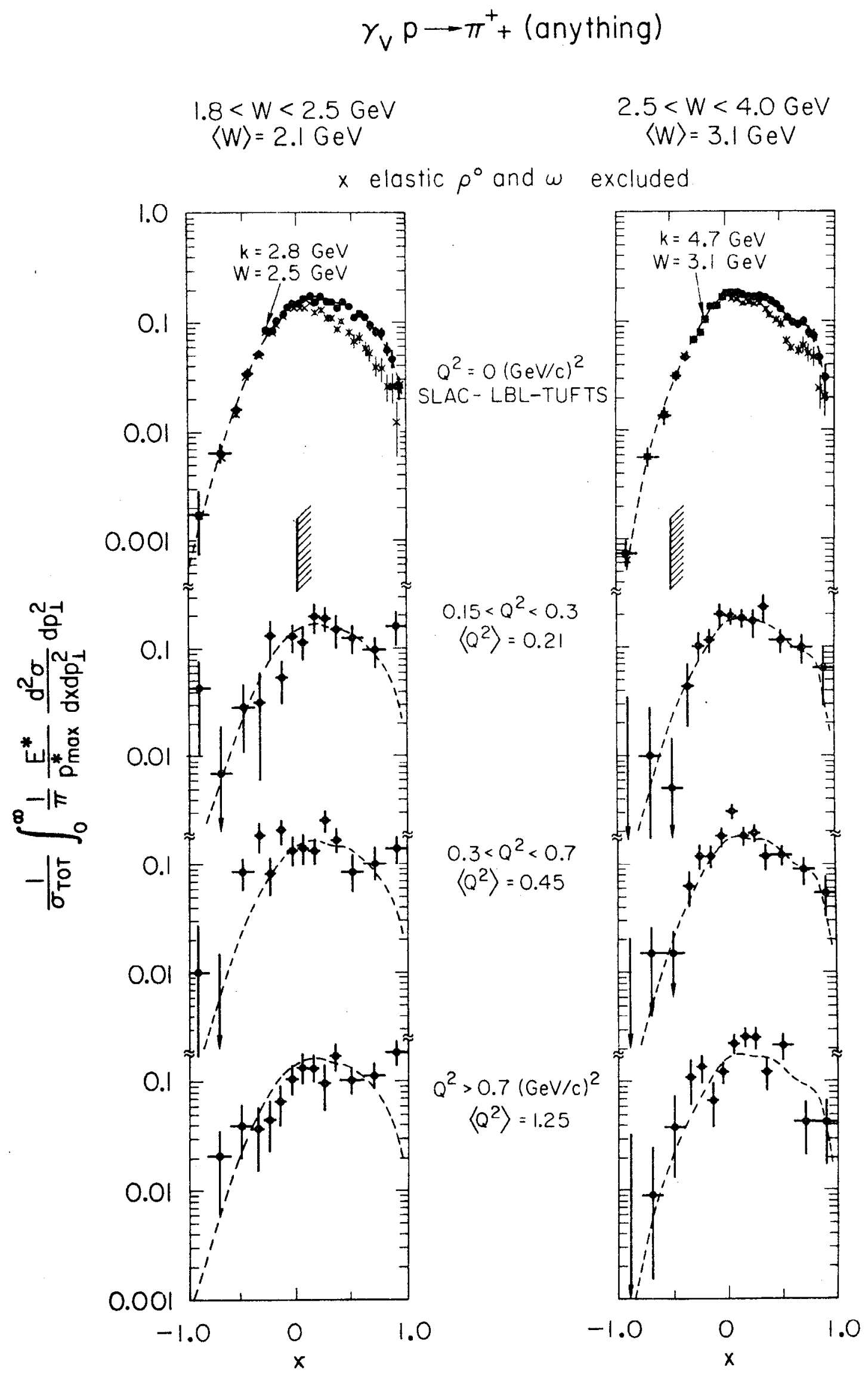

Fig. 13 


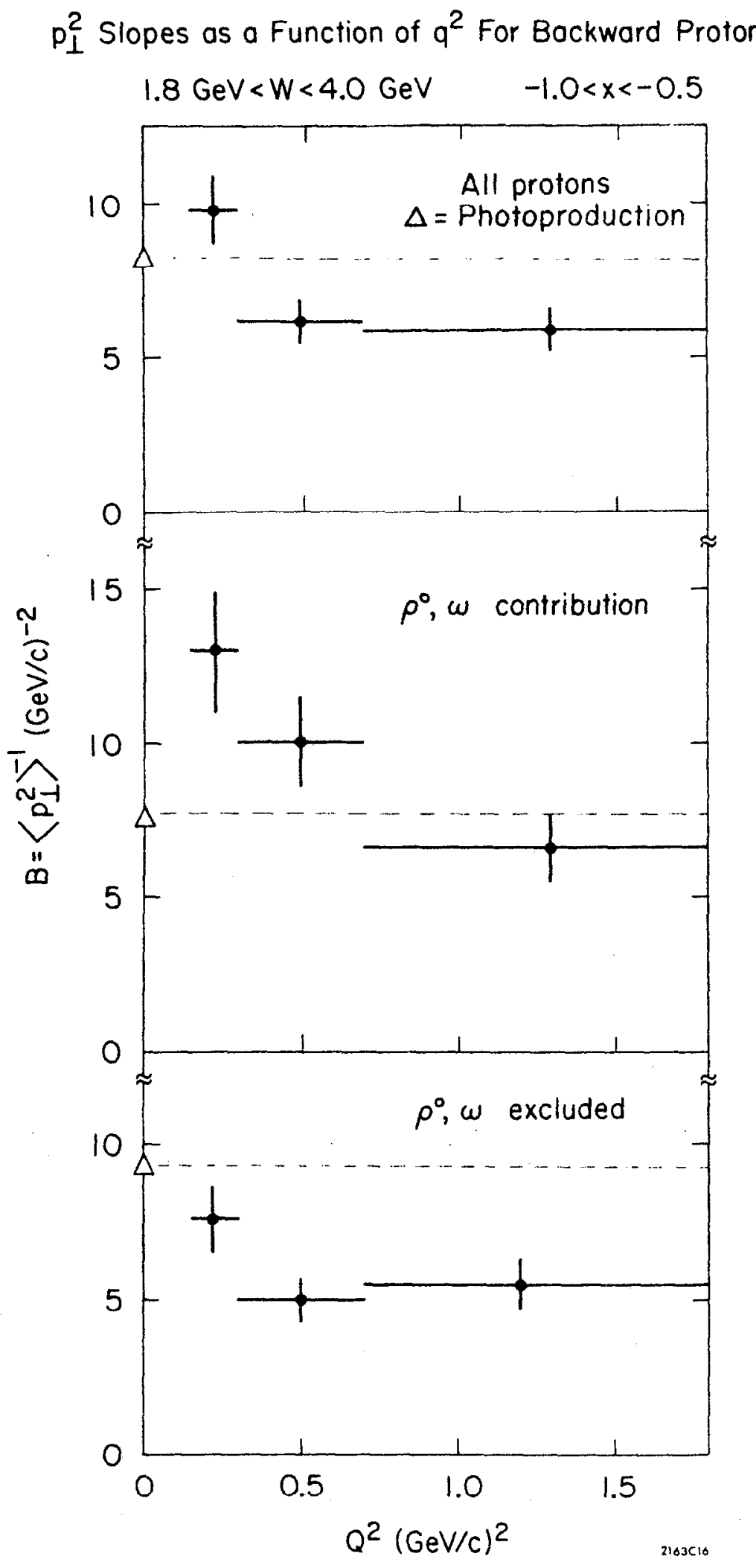

Fig. 14 
\pm Inclusive Charge Ratio $V s Q^{2}$

$1.8 \leq W \leq 4.0,\langle W\rangle=2.6 \mathrm{Gev}$

$\Delta=$ Photoproduction

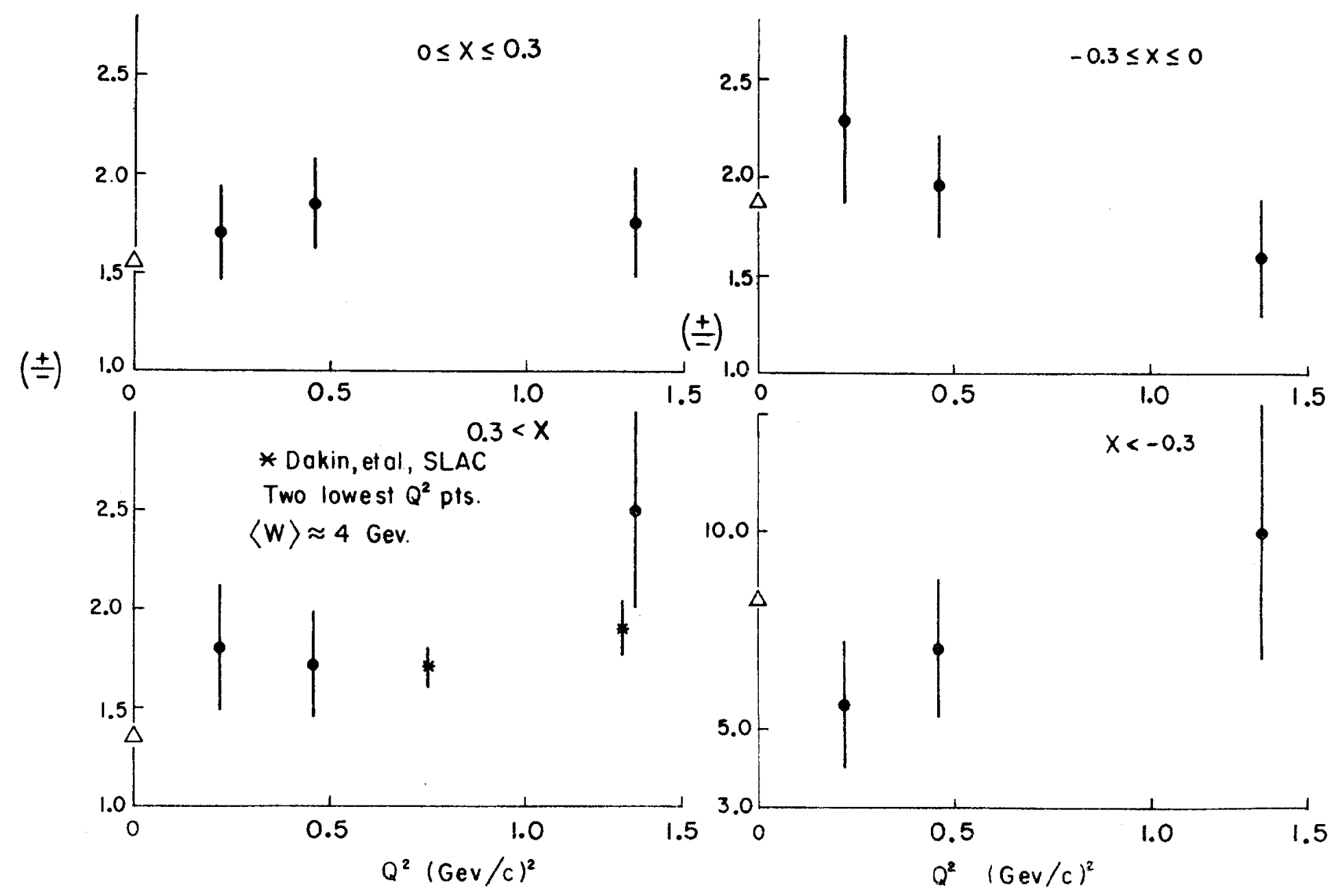

